QUARTERLY OF APPLIED MATHEMATICS

VOLUME LXIX, NUMBER 1

MARCH 2011, PAGES 177-202

S 0033-569X(2011)01221-6

Article electronically published on January 19, 2011

\title{
INSTANTANEOUS SHOCK LOCATION AND ONE-DIMENSIONAL NONLINEAR STABILITY OF VISCOUS SHOCK WAVES
}

\author{
BY \\ KEVIN ZUMBRUN \\ Department of Mathematics, Indiana University, Bloomington, Indiana 47405
}

\begin{abstract}
We illustrate in a simple setting the instantaneous shock tracking approach to stability of viscous conservation laws introduced by Howard, Mascia, and Zumbrun. This involves a choice of the definition of instantaneous location of a viscous shock. We show that this choice is time-asymptotically equivalent both to the natural choice of least-squares fit pointed out by Goodman and to a simple phase condition used by Guès, Métivier, Williams, and Zumbrun in other contexts. More generally, we show that it is asymptotically equivalent to any location defined by a localized projection.
\end{abstract}

1. Introduction. In this paper, we illustrate in the simple and concrete setting of Burgers equation the argument for nonlinear stability of viscous shock waves developed for general systems of conservation laws in [Z1, MaZ2, MaZ3, MaZ4, based on instantaneous tracking of the location of the perturbed viscous shock wave. The advantage of Burgers equation is that the linearized equations may be solved explicitly by a linearized Hopf-Cole transformation, thus isolating the nonlinear issues we wish to discuss. This same example was given in [Z1]; here we expand a bit the surrounding discussion, reexamining the question of what is a reasonable or natural definition of the instantaneous location of a perturbed viscous shock wave and adding a discussion of the small-amplitude limit.

Using the purely operational but analytically tractable definition of [Z1, MaZ2, MaZ4] as a tool for comparison, we show that any definition based on localized projection is time-asymptotically equivalent to any other and to the definition of [Z1, MaZ2, MaZ4]; see Appendix [C, and especially Remarks C.3 C.4. Moreover, any of these may be used as the basis of a nonlinear stability argument. This includes in particular both the natural definition by least squares fit pointed out early on by Goodman [G] and, in the

Received September 28, 2009.

2010 Mathematics Subject Classification. Primary 35B35.

Key words and phrases. Viscous shock waves; nonlinear stability; pointwise Green function bounds.

The author's research was partially supported under NSF grants no. DMS-0300487 and DMS-0801745.

E-mail address: kzumbrun@indiana.edu

(C) 2011 Brown University

Reverts to public domain 28 years from publication 
limit of infinite localization (to a single point), a very simple definition based on a phase condition, introduced by Guès, Métivier, Williams, and Zumbrun GMWZ.

Our analysis is intended for the nonspecialist. It is brief and self-contained except for standard linear and short-time parabolic existence theory. Though we restrict here for simplicity to the scalar Burgers case, our arguments and conclusions extend in straightforward fashion to the general system case [Z1, MaZ2, MaZ4, once there are established the requisite bounds on the linearized solution operator. This separate, and in general difficult, problem has been treated in $\mathrm{ZH}, \mathrm{MaZ3}, \mathrm{Z2}$; see Remark 3.4 and the discussion of Section 4. Our purpose here is, rather, to isolate the issues connected with viscous shock-tracking and the nonlinear iteration argument by restricting to a case where the linearized bounds are available by an exact solution formula.

1.1. Problem and equations. Consider the scalar viscous conservation law

$$
u_{t}+f(u)_{x}=u_{x x},
$$

$u=u(x, t) \in \mathbb{R}, x \in \mathbb{R}, t \in \mathbb{R}^{+}$, with

$$
f(u)=u^{2} / 2 .
$$

Equation (1.1) serves as a simple model for gas dynamics, traffic flow, or shallow-water waves, where $u$ represents the density of some conserved quantity and $f$ its flux through a fixed point $x$. With the choice of flux (1.2), (1.1) becomes Burgers equation, the prototypical example of a scalar viscous conservation law. The behavior for other (convex) fluxes is qualitatively similar.

We investigate the question of nonlinear stability of solutions $u=\bar{u}$, that is, whether a perturbation $\tilde{u}=\bar{u}+u$ remains close to $\bar{u}$ in some norm for initial perturbations $\left.u\right|_{t=0}=$ $\left.(\tilde{u}-\bar{u})\right|_{t=0}$ sufficiently small in some (possibly different) norm: more specifically, nonlinear asymptotic stability, that is, whether $\tilde{u}$ both remains near and converges to $\bar{u}$ as $t \rightarrow$ $+\infty$ for initial perturbations sufficiently small. Since the equation (1.1) is translationinvariant, we must when relevant (specifically, when translates of $\bar{u}$ are not equal to $\bar{u})$ adjust the second notion to that of nonlinear asymptotic orbital stability, defined as nonlinear stability together with convergence as $t \rightarrow+\infty$ to the set of translates of $\bar{u}$, as discussed further below.

1.2. Constant and traveling-wave solutions. An obvious class of solutions of (1.1) is the set of constant solutions $\bar{u} \equiv a, a \in \mathbb{R}$. A second class of solutions is the viscous shock waves, or smooth traveling-wave solutions

$$
u(x, t)=\bar{u}(x-s t), \quad \lim _{x \rightarrow \pm \infty} \bar{u}(x)=u_{ \pm},
$$

$s$ constant, connecting constant endstates $u_{ \pm}$. If $s=0$, they are equilibria, or stationary waves of the associated evolution equation (1.1). A traveling wave may always be converted to a standing wave by the change of coordinates $x \rightarrow x-s t$ to a frame moving with the same speed $s$.

Observing that $\partial_{t} \bar{u}(x-s t)=-s \bar{u}^{\prime}, \partial_{x} \bar{u}(x-s t)=\bar{u}^{\prime}$, and $\partial_{x}^{2} \bar{u}(x-s t)=\bar{u}^{\prime \prime}$, we obtain for a solution (1.3) the profile equation $-s \bar{u}^{\prime}+f(\bar{u})^{\prime}=\bar{u}^{\prime \prime}$. Integrating from $-\infty$ to $x$ reduces this to a first-order equation

$$
\bar{u}^{\prime}=(f(\bar{u})-s \bar{u})-\left(f\left(u_{-}\right)-s u_{-}\right) .
$$


Taking, for definiteness, $s=0$ and $u_{-}=1$, we obtain $\bar{u}^{\prime}=(1 / 2)\left(\bar{u}^{2}-1\right)$, which has the explicit solution

$$
\bar{u}(x)=-\tanh (x / 2)
$$

connecting endstates $u_{ \pm}=\mp 1$. This is the unique solution up to translation in $x$ connecting that particular pair of endstates. Other endstates and speeds also lead to tanh profiles, as may be seen by invariances of Burgers equation; thus, we may without loss of generality restrict to this specific case.

2. Stability of constant solutions. To indicate the basic approach, let us first consider the stability of a constant solution

$$
\bar{u} \equiv a, \quad a \in \mathbb{R}
$$

of (1.1). Letting $\tilde{u}$ be a second solution of (1.1), and defining the perturbation $u:=\tilde{u}-\bar{u}$, we obtain after a brief computation the perturbation equation

$$
u_{t}-L u=N(u)_{x},
$$

where $L u:=u_{x x}-a u_{x}$ is the linearization of $u_{x x}-f(u)_{x}$ about the solution $\bar{u} \equiv a$, and $N(u):=-u^{2} / 2$ is a remainder of quadratic order.

2.1. Linear solution operator. The homogeneous linearized equations $v_{t}-L v=0$ may be recognized as a convected heat equation

$$
v_{t}+a v_{x}=v_{x x},\left.\quad v\right|_{t=0}=f .
$$

This admits an exact solution

$$
e^{L t} f=\int_{-\infty}^{+\infty} G_{0}(x, t ; y) f(y) d y
$$

where

$$
G_{0}(x, t ; y):=e^{L t} \delta_{y}(x)=\frac{e^{-\frac{|x-y-a t|^{2}}{4 t}}}{\sqrt{4 \pi t}}
$$

is the Green function for (2.3), a convected heat kernel. This yields in particular a unique classical solution $v \in C^{0}\left(t \geq 0 ; L^{p}(x)\right) \cap C^{2}(t>0, x)$ for each $f \in L^{p}$.

Easy scaling arguments yield, for $1 \leq p \leq \infty$,

$$
\begin{aligned}
\left|G_{0}(\cdot, t ; y)\right|_{L^{p}(x)} & =\left|G_{0}(x, t ; \cdot)\right|_{L^{p}(y)}=C_{p} t^{-\frac{1}{2}(1-1 / p)}, \\
\left|G_{0, y}(\cdot, t ; y)\right|_{L^{p}(x)} & =\left|G_{0, y}(x, t ; \cdot)\right|_{L^{p}(y)}=C_{p}^{\prime} t^{-\frac{1}{2}(1-1 / p)-\frac{1}{2}},
\end{aligned}
$$

for some constants $C_{p}, C_{p}^{\prime}>0$. From (2.6), we readily obtain the following linearized estimates (standard heat kernel bounds).

Lemma 2.1. For some $C>0$, all $t>0$,

$$
\begin{gathered}
\left|\int_{-\infty}^{+\infty} G_{0}(x, t ; y) f(y) d y\right|_{L^{p}} \leq C t^{-\frac{1}{2}(1-1 / p)}|f|_{L^{1}(x)}, \\
\left|\int_{-\infty}^{+\infty} G_{0, y}(x, t ; y) f(y) d y\right|_{L^{p}} \leq C t^{-\frac{1}{2}(1-1 / p)-\frac{1}{2}}|f|_{L^{1}(x)} .
\end{gathered}
$$


Proof. Applying the triangle inequality together with (2.6), we obtain

$$
\left|\int_{-\infty}^{+\infty} G_{0}(x, t ; y) f(y) d y\right|_{L^{p}(x)} \leq \int_{-\infty}^{+\infty}\left|G_{0}(\cdot, t ; y)\right|_{L^{p}(x)}|f(y)| d y=C_{p} t^{-\frac{1}{2}(1-1 / p)}|f|_{L^{1}} .
$$

The proof of the second inequality is similar.

Lemma 2.2. For some $C>0$, all $t>0$,

$$
\left|\int_{-\infty}^{+\infty} G_{0}(x, t ; y) f(y) d y\right|_{L^{p}(x)} \leq C|f|_{L^{p}}, \quad\left|\int_{-\infty}^{+\infty} G_{0, y}(x, t ; y) f(y) d y\right|_{L^{p}(x)} \leq C t^{-\frac{1}{2}}|f|_{L^{p}} .
$$

Proof. Noting that $G_{0}(x, t ; y)=G_{0}(x-y, t ; 0)$, so that (2.4) is a convolution, we may rewrite $\int_{-\infty}^{+\infty} G_{0}(x, t ; y) f(y) d y$ as $\int_{-\infty}^{+\infty} G_{0}(z, t ; 0) f(x-z) d z$ with $z:=x-y$. Applying the triangle inequality and (2.6), we obtain

$$
\left|\int_{-\infty}^{+\infty} G_{0}(x, t ; y) f(y) d y\right|_{L^{p}(x)} \leq \int_{-\infty}^{+\infty}\left|G_{0}(z, t ; 0)\right||f|_{L^{p}} d z=C_{1}|f|_{L^{p}}
$$

The proof of the second inequality is similar.

2.2. Integral representation. From the homogeneous linearized solution formula (2.4), we obtain by variation of constants/Duhamel's formula a solution for the inhomogeneous linearized equations

$$
v_{t}-L v=g,\left.\quad v\right|_{t=0}=f
$$

of $v=e^{L t} f+\int_{0}^{t} e^{L(t-s)} g(s) d s$, or

$$
v(x, t)=\int_{-\infty}^{+\infty} G_{0}(x, t ; y) f(y) d y+\int_{0}^{t} \int_{-\infty}^{+\infty} G_{0}(x, t-s ; y) g(y, s) d y d s,
$$

yielding a unique $C^{0}\left(t \geq 0 ; L^{p}(x)\right) \cap C^{2}(t>0 ; x)$ solution $v$ for $f \in L^{p}$ and $g \in$ $L^{1}\left(t ; W^{-1, p}(x)\right)$.

2.3. Nonlinear iteration. Returning now to the nonlinear problem (2.2), we have, setting $g=N(u)_{x}$ in (2.9), the representation $u(x, t)=\int_{-\infty}^{+\infty} G_{0}(x, t ; y) u_{o}(y) d y+$ $\int_{0}^{t} \int_{-\infty}^{+\infty} G_{0}(x, t-s ; y) N(u(y, s))_{y} d y d s$, or, integrating the last term by parts,

$$
u(x, t)=\int_{-\infty}^{+\infty} G_{0}(x, t ; y) u_{0}(y) d y-\int_{0}^{t} \int_{-\infty}^{+\infty} G_{0, y}(x, t-s ; y) N(u(y, s)) d y d s,
$$

valid so long as the solution $u$ exists and remains sufficiently smooth that (2.11) gives the unique solution to the associated inhomogeneous problem, in particular for $u_{0} \in L^{p} \cap L^{\infty}$ and $u$ in $C^{0}\left(t \geq 0 ; L^{p} \cap L^{\infty}\right) \cap C^{2}(t>0, x)$, any $p \geq 1$.

On the other hand, standard short-time existence theory (proved, e.g., by contractionmapping using a similar representation with shifted initial time) yields existence of a $C^{0}\left(t \geq 0 ; L^{p} \cap L^{\infty}(x)\right) \cap C^{2}(t>0, x)$ solution so long as $|u|_{L^{p} \cap L^{\infty}}$ remains bounded.

Now define

$$
\zeta(t):=\sup _{0 \leq s \leq t, 1 \leq p \leq \infty}|u|_{L^{p}}(s)(1+s)^{\frac{1}{2}(1-1 / p)} .
$$

Lemma 2.3. For all $t \geq 0$ for which $\zeta(t)$ is finite, some $C>0$, and $E_{0}:=\left|u_{0}\right|_{L^{1} \cap L^{\infty}}$,

$$
\zeta(t) \leq C\left(E_{0}+\zeta(t)^{2}\right)
$$


Proof. Noting, by quadratic dependence $N(u)=O\left(|u|^{2}\right)$ and the definition (2.12) of $\zeta$, that

$$
\begin{aligned}
& |N(u)|_{L^{1}} \leq C|u|_{L^{2}}^{2} \leq C \zeta(t)^{2}(1+t)^{-\frac{1}{2}}, \\
& |N(u)|_{L^{p}} \leq C|u|_{L^{p}}|u|_{L^{\infty}} \leq C \zeta(t)^{2}(1+t)^{-\frac{1}{2}(1-1 / p)-\frac{1}{2}},
\end{aligned}
$$

we obtain, applying Lemmas 2.12 .2 to representation (2.11), the estimate

$$
\begin{aligned}
|u(\cdot, t)|_{L^{p}(x)} \leq & \left|\int_{-\infty}^{+\infty} G_{0}(x, t ; y) u_{0}(y) d y\right|_{L^{p}(x)} \\
& +\left|\int_{0}^{t / 2} \int_{-\infty}^{+\infty} G_{0, y}(x, t-s ; y) N(u(y, s)) d y d s\right|_{L^{p}(x)} \\
& +\left|\int_{t / 2}^{t} \int_{-\infty}^{+\infty} G_{0, y}(x, t-s ; y) N(u(y, s)) d y d s\right|_{L^{p}(x)} \\
\leq & C(1+t)^{-\frac{1}{2}(1-1 / p)} E_{0}+C \zeta(t)^{2} \int_{0}^{t / 2}(t-s)^{-\frac{1}{2}(1-1 / p)-1 / 2}(1+s)^{-\frac{1}{2}} d s \\
& +C \zeta(t)^{2} \int_{t / 2}^{t}(t-s)^{-\frac{1}{2}}(1+s)^{-\frac{1}{2}(1-1 / p)-\frac{1}{2}} d s \\
\leq & C\left(E_{0}+\zeta(t)^{2}\right)(1+t)^{-\frac{1}{2}(1-1 / p)} .
\end{aligned}
$$

Rearranging, we obtain (2.13).

Corollary 2.4 (Stability of constant solutions). Constant solutions $\bar{u} \equiv a$ are nonlinearly stable in $L^{1} \cap L^{\infty}$ and nonlinearly asympotically stable in $L^{p}, p>1$, with respect to initial perturbations $u_{0}$ that are sufficiently small in $L^{1} \cap L^{\infty}$. More precisely, for some $C>0$,

$$
|\tilde{u}-\bar{u}|_{L^{p}}(t) \leq\left. C(1+t)^{-\frac{1}{2}(1-1 / p)}|\tilde{u}-\bar{u}|_{L^{1} \cap L^{\infty}}\right|_{t=0}
$$

for all $t \geq 0,1 \leq p \leq \infty$, for solutions $\tilde{u}$ of (1.1) with $\left.|\tilde{u}-\bar{u}|_{L^{1} \cap L^{\infty}}\right|_{t=0}$ sufficiently small.

Proof. ("Continuous induction") By Lemma 2.3, $\zeta(t) \leq C\left(E_{0}+\zeta(t)^{2}\right)$ for

$$
E_{0}:=\left.|\tilde{u}-\bar{u}|_{L^{1} \cap L^{\infty}}\right|_{t=0}
$$

Taking $E_{0}<\frac{1}{4 C^{2}}$, we have therefore that $\zeta(t)<2 C E_{0}$ whenever $\zeta(t) \leq 2 C E_{0}$, and so the set of $t \geq 0$ for which $\zeta(t)<2 C E_{0}$ is equal to the set of $t \geq 0$ for which $\zeta(t) \leq 2 C E_{0}$. Recalling, by the cited standard short-time existence theory, that $\zeta$ is continuous wherever it is finite, we find, therefore, that the set of $t \geq 0$ for which $\zeta(t)<2 C E_{0}$ is both open and closed. Taking without loss of generality $C>1 / 2$, so that $t=0$ is contained in this set, we have that the set is nonempty. It follows that $\zeta(t)<2 C E_{0}$ for all $t \geq 0$, yielding (2.16) by definitions (2.12) and (2.17).

REMARK 2.5. The rate of decay (2.16) is that of a heat kernel; that is, the mechanism for stability is diffusive only.

3. Stability of viscous shock solutions. We turn now to the stability of viscous shock solutions of (1.1), without loss of generality, restricting to the case

$$
\bar{u}(x)=-\tanh (x / 2)
$$


described in (1.5). Letting $\tilde{u}$ as before be a second solution of (1.1), define the perturbation

$$
u(x, t):=\tilde{u}(x+\alpha(t), t)-\bar{u}(x)
$$

as the difference between a translate of $\tilde{u}$ and the background wave $\bar{u}$, where the translation $\alpha(t)$ is to be determined later.

This yields after a brief computation the perturbation equation

$$
u_{t}-L u=N(u)_{x}+\dot{\alpha}(t)\left(\bar{u}_{x}+u_{x}\right),
$$

where $L u:=u_{x x}-(a(x) u)_{x}$ is the linearization of $u_{x x}-f(u)_{x}$ about the solution $\bar{u}=$ $-\tanh (x / 2), a(x):=d f(\bar{u})(x)=\bar{u}(x)$, and $N(u):=-u^{2} / 2$ is the same remainder of quadratic order as in the constant-coefficient case.

3.1. Linear solution operator/decomposition of the Green function. The homogeneous linearized equation

$$
v_{t}-L v=v_{t}+(a(x) v)_{x}-v_{x x}=0,\left.\quad v\right|_{t=0}=f
$$

can be solved explicitly by a linearized Hopf-Cole transformation [S, $\mathrm{N}, \mathrm{Z3}$, GSZ] to give an exact solution formula

$$
e^{L t} f=\int_{-\infty}^{+\infty} G(x, t ; y) f(y) d y
$$

where

$$
\begin{aligned}
G(x, t ; y):=e^{L t} \delta_{y}(x)= & \bar{u}^{\prime}(x)\left(\frac{1}{2}\right)\left(\operatorname{errfn}\left(\frac{x-y-t}{\sqrt{4 t}}\right)-\operatorname{errfn}\left(\frac{x-y+t}{\sqrt{4 t}}\right)\right) \\
& +\left(\left(\frac{e^{-\frac{x}{2}}}{e^{\frac{x}{2}}+e^{-\frac{x}{2}}}\right) \frac{e^{-\frac{(x-y-t)^{2}}{4 t}}}{\sqrt{4 \pi t}}+\left(\frac{e^{\frac{x}{2}}}{e^{\frac{x}{2}}+e^{-\frac{x}{2}}}\right) \frac{e^{-\frac{(x-y+t)^{2}}{4 t}}}{\sqrt{4 \pi t}}\right)
\end{aligned}
$$

is the Green function for (3.3) and $\operatorname{errfn}(z):=\frac{1}{\sqrt{\pi}} \int_{-\infty}^{z} e^{-\xi^{2}} d \xi$.

Following the approach of [Z1, MaZ2, MaZ4], now decompose

$$
G(x, t ; y):=E(x, t ; y)+S(x, t ; y)+R(x, t ; y),
$$

where

$$
\begin{gathered}
E(x, t ; y):=\bar{u}^{\prime}(x) e(y, t), \quad e(y, t):=\left(\frac{1}{2}\right)\left(\operatorname{errfn}\left(\frac{-y-t}{\sqrt{4 t}}\right)-\operatorname{errfn}\left(\frac{-y+t}{\sqrt{4 t}}\right)\right) \\
S(x, t ; y):=\left(\left(\frac{e^{-\frac{x}{2}}}{e^{\frac{x}{2}}+e^{-\frac{x}{2}}}\right) \frac{e^{-\frac{(x-y-t)^{2}}{4 t}}}{\sqrt{4 \pi t}}+\left(\frac{e^{\frac{x}{2}}}{e^{\frac{x}{2}}+e^{-\frac{x}{2}}}\right) \frac{e^{-\frac{(x-y+t)^{2}}{4 t}}}{\sqrt{4 \pi t}}\right)
\end{gathered}
$$

and

$$
\begin{aligned}
R(x, t ; y):= & \bar{u}^{\prime}(x)\left(\frac{1}{2}\right)\left(\operatorname{errfn}\left(\frac{x-y-t}{\sqrt{4 t}}\right)-\operatorname{errfn}\left(\frac{-y-t}{\sqrt{4 t}}\right)\right) \\
& -\bar{u}^{\prime}(x)\left(\frac{1}{2}\right)\left(\operatorname{errfn}\left(\frac{x-y+t}{\sqrt{4 t}}\right)-\operatorname{errfn}\left(\frac{-y+t}{\sqrt{4 t}}\right)\right) .
\end{aligned}
$$

Here, the "excited term" $E$ represents the nondecaying part of the linearized solution $v$, involving the zero eigenfunction $L \bar{u}^{\prime}=0$ associated with instantaneous translation of the background wave, the "scattering term" $S$ comprises Gaussian signals convected along hyperbolic characteristics, and the "remainder term" $R$ a faster-decaying residual. 
A straightforward calculation gives

$$
\begin{aligned}
\mid R(x, t ; y \mid & \leq C|x|\left|\bar{u}^{\prime}(x)\right| \int_{0}^{1}\left(\frac{e^{-\frac{(\xi x-y-t)^{2}}{4 t}}}{\sqrt{4 \pi t}}-\frac{e^{-\frac{(\xi x-y+t)^{2}}{4 t}}}{\sqrt{4 \pi t}}\right) d \xi \\
& \leq C e^{-\theta|x|} \int_{0}^{1}\left(\frac{e^{-\frac{(\xi x-y-t)^{2}}{4 t}}}{\sqrt{4 \pi t}}+\frac{e^{-\frac{(\xi x-y+t)^{2}}{4 t}}}{\sqrt{4 \pi t}}\right) d \xi
\end{aligned}
$$

$\theta>0$, showing that $R$, as the product of an exponentially decaying term and the sum of convected Gaussians, is indeed faster-decaying than either $E$ or $S$.

Lemma 3.1. For some $C>0, \theta>0$, all $t>0$,

$$
\begin{gathered}
\mid R\left(x, t ; y \mid \leq C e^{-\theta|x| / C}\left(\frac{e^{-\frac{(x-y-t)^{2}}{4 C t}}}{\sqrt{t}}+\frac{e^{-\frac{(x-y+t)^{2}}{4 C t}}}{\sqrt{t}}\right)+C e^{-\theta(|x-y|+t)},\right. \\
\mid R_{y}\left(x, t ; y \mid \leq C e^{-\theta|x| / C}\left(\frac{e^{-\frac{(x-y-t)^{2}}{4 C t}}}{t}+\frac{e^{-\frac{(x-y+t)^{2}}{4 C t}}}{t}\right)+\frac{C e^{-\theta(|x-y|+t)}}{\sqrt{t}} .\right.
\end{gathered}
$$

Proof. Applying the Cauchy-Schwarz inequality in the argument of the exponential, we find readily that

$$
e^{-\theta|x| / 2}\left(e^{-\frac{(\xi x-y-t)^{2}}{4 t}}+e^{-\frac{(\xi x-y+t)^{2}}{4 t}}\right) \leq e^{-\theta|x| / C}\left(e^{-\frac{(x-y-t)^{2}}{4 C t}}+e^{-\frac{(x-y+t)^{2}}{4 C t}}\right)
$$

for $|x| \leq M t$ and $C>0$ sufficiently large; hence (3.10) implies (3.11)(i). For $|y| \gg|x|+|t|$, (3.12) holds trivially, likewise giving (3.11)(i). In both of these cases, the left-hand side is bounded by the first, Gaussian, term alone on the right-hand side. In the remaining case $|x| \gg t$ and $|y| \leq M|x|$, we have for $C>0$ sufficiently large that $e^{-\theta|x|} \leq e^{-(\theta / C)(|x-y|+t)}$, from which we find directly from (3.9) that the left-hand side of (3.11) (i) is bounded by the final term on the right-hand side.

Similar computations yield (3.11) (ii).

REMARK 3.2. The excited term $E$ converges as $t \rightarrow+\infty$ to $\bar{u}^{\prime}(x)$ times

$$
-\sigma(+\infty):=\int_{-\infty}^{+\infty} e(y,+\infty) f(y) d y=(1 / 2) \int_{-\infty}^{+\infty} f(y) d y
$$

the time-asymptotic state of the linearized equations (3.3) determined by conservation of mass (equals total integral $\int_{-\infty}^{+\infty} v(x, t) d x$ ). Note that $\bar{u}^{\prime}(x)$ corresponds to infinitesimal translation of the background wave $\bar{u}(x)$; hence a linear time-asymptotic state $-\sigma \bar{u}^{\prime}(x)$ corresponds roughly to a steady-state perturbation $\bar{u}(x-\sigma)-\bar{u}(x)$ consisting of a shift, or translation, $\sigma$ of the background wave. The term $\sigma(t):=-\int_{-\infty}^{+\infty} e(y, t) f(y) d y$ thus measures, at a linearized level, the shift in location of the shock at time $t$, or "instantaneous shock shift". This refines the picture of behavior given by the time-asymptotic shock shift $\sigma(+\infty)$. 
Proposition 3.3. The Green function $G$ decomposes as $G=E+\tilde{G}, E=\bar{u}^{\prime}(x) e(y, t)$, where, for some $C>0$, all $t>0$,

$$
\begin{gathered}
\left|\int_{-\infty}^{+\infty} \tilde{G}(x, t ; y) f(y) d y\right|_{L^{p}(x)} \leq C t^{-\frac{1}{2}(1-1 / p)}|f|_{L^{1}} \\
\left|\int_{-\infty}^{+\infty} \tilde{G}_{y}(x, t ; y) f(y) d y\right|_{L^{p}(x)} \leq C t^{-\frac{1}{2}(1-1 / p)-\frac{1}{2}}|f|_{L^{1}} \\
\left|\int_{-\infty}^{+\infty} \tilde{G}(x, t ; y) f(y) d y\right|_{L^{p}(x)} \leq C|f|_{L^{p}}, \quad\left|\int_{-\infty}^{+\infty} \tilde{G}_{y}(x, t ; y) f(y) d y\right|_{L^{p}(x)} \leq C t^{-\frac{1}{2}}|f|_{L^{p}} \\
\left|\int_{-\infty}^{+\infty} e_{t}(y, t) f(y) d y\right| \leq C t^{-\frac{1}{2}}|f|_{L^{1}}, \quad\left|\int_{-\infty}^{+\infty} e_{t y}(y, t) f(y) d y\right| \leq C t^{-1}|f|_{L^{1}} \\
\left|\int_{-\infty}^{+\infty} e_{t}(y, t) f(y) d y\right| \leq C|f|_{L^{\infty}}, \quad\left|\int_{-\infty}^{+\infty} e_{y t}(y, t) f(y) d y\right| \leq C t^{-\frac{1}{2}}|f|_{L^{\infty}}
\end{gathered}
$$

and

$$
\left|\int_{-\infty}^{+\infty} e(y, t) f(y) d y\right| \leq C|f|_{L^{1}}, \quad\left|\int_{-\infty}^{+\infty} e_{y}(y, t) f(y) d y\right| \leq C t^{-1 / 2}|f|_{L^{1}} .
$$

Proof. Defining $\tilde{G}:=R+S$, we have the decomposition $G=E+\tilde{G}$. By (3.8) and estimate (3.11), $\tilde{G}$ and $\tilde{G}_{y}$ obey essentially the same bounds as $G$ and $G_{y}$ in the constant-coefficient case (2.5), up to a harmless exponential error (the final terms on the right-hand sides of (3.11) ). Thus, bounds (3.13) and (3.14) follow by the same argument used to prove (2.7) and (2.8). By (3.7), $\left|e_{t}\right|$ and $\left|e_{y t}\right|$ satisfy essentially the same bounds as $\sup _{x}|\tilde{G}|$ and $\sup _{x}\left|\tilde{G}_{y}\right|$; hence (3.15) and (3.16) follow again from this same argument in case $p=\infty$, which amounts to Hölder's inequality together with $L^{p}$ bounds on $e$ and derivatives (see Lemma D.1. Appendix D.1 for a careful derivation of these $L^{p}$ bounds). Finally, (3.17) follows by $|e| \leq C,\left|e_{y}\right| \leq C t^{-1 / 2}$ using the triangle inequality.

Remark 3.4. The apparently special Proposition 3.3 in fact holds for viscous shock waves of general strictly parabolic systems provided that the shock satisfies a generalized spectral stability, i.e., Evans function, condition [Z1, Z4, MaZ3]. Indeed, there is a parallel decomposition of the Green function as the sum of terms $E, S$, and $R$ with pointwise descriptions generalizing those of (3.7), (3.8), (3.11). Similar bounds hold for Evans stable shocks of general hyperbolic-parabolic systems $u_{t}+f(u)_{x}=\left(B(u) u_{x}\right)_{x}$ with $B=\left(\begin{array}{cc}0 & 0 \\ b_{2} & b_{2}\end{array}\right)$, under mild compatibility conditions on $d f\left(u_{ \pm}\right)$and $B\left(u_{ \pm}\right)$satisfied for standard physical equations such as the Navier-Stokes equations of compressible gasdynamics or MHD with van der Waals equation of state, or the equations of viscoelasticity MaZ3, Z4. Scalar shock waves are always spectrally stable, by the maximum principle; hence, the stability condition does not make itself apparent for Burgers equation.

In the derivation of bounds by inverse Laplace transform estimates, the terms $E$ and $S$ arise in a very natural way as leading terms of a low-frequency "scattering" expansion [MaZ3, Z2] of the resolvent kernel about frequency $\lambda=0$, without the need to rearrange terms as done here in the Burgers case. See Section 2, BeSZ, for a particulary clear discussion of the method from a more general point of view. Indeed, the decomposition 
of $G$ into $E$ and $\tilde{G}$ was suggested from the inverse Laplace transform point of view [ZH, Z1, MaZ3]. Here, for pedagogical purposes, we have imposed this structure by force on the explicit Green function given by a Hopf-Cole transformation in order to demonstrate clearly the approach.

3.2. Integral representation/ $\alpha$-evolution scheme. Recalling that $\bar{u}^{\prime}(x)$ is a stationary solution of the linearized equations $u_{t}=L u$, so that $L \bar{u}_{x}=0$, or

$$
\int_{-\infty}^{\infty} G(x, t ; y) \bar{u}_{x}(y) d y=e^{L t} \bar{u}_{x}(x)=\bar{u}_{x}(x),
$$

we have, applying Duhamel's principle to (3.2),

$$
\begin{aligned}
u(x, t)= & \int_{-\infty}^{\infty} G(x, t ; y) u_{0}(y) d y \\
& -\int_{0}^{t} \int_{-\infty}^{\infty} G_{y}(x, t-s ; y)(N(u)+\dot{\alpha} u)(y, s) d y d s+(\alpha(t)-\alpha(0)) \bar{u}^{\prime}(x) .
\end{aligned}
$$

Defining $\alpha$ implicitly as

$$
\begin{aligned}
\alpha(t)= & -\int_{-\infty}^{\infty} e(y, t) u_{0}(y) d y \\
& +\int_{0}^{t} \int_{-\infty}^{+\infty} e_{y}(y, t-s)(N(u)+\dot{\alpha} u)(y, s) d y d s,
\end{aligned}
$$

following [ZH, Z4, MaZ2, MaZ3, where $e$ is defined as in (3.7), and substituting in (3.18) the decomposition $G=\bar{u}^{\prime}(x) e+\tilde{G}$ of Proposition 3.3. we obtain the integral representation

$$
\begin{aligned}
u(x, t)= & \int_{-\infty}^{\infty} \tilde{G}(x, t ; y) u_{0}(y) d y \\
& -\int_{0}^{t} \int_{-\infty}^{\infty} \tilde{G}_{y}(x, t-s ; y)(N(u)+\dot{\alpha} u)(y, s) d y d s,
\end{aligned}
$$

and, differentiating (3.19) with respect to $t$, and observing that $e_{y}(y, s) \rightarrow 0$ as $s \rightarrow 0$, as the difference of approaching heat kernels,

$$
\begin{aligned}
\dot{\alpha}(t)= & -\int_{-\infty}^{\infty} e_{t}(y, t) u_{0}(y) d y \\
& +\int_{0}^{t} \int_{-\infty}^{+\infty} e_{y t}(y, t-s)(N(u)+\dot{\alpha} u)(y, s) d y d s .
\end{aligned}
$$

(Note: in obtaining (3.20), we have used the fact that $e(y, 0) \equiv 0$ to conclude that $\alpha(0)=0$.)

Equations (3.20), (3.21) together form a complete system in the variables $(u, \dot{\alpha})$, from the solution of which we may afterward recover the shift $\alpha$ via (3.19). From the original differential equation (3.2) together with (3.21), we readily obtain short-time existence and continuity with respect to $t$ of solutions $(u, \dot{\alpha}) \in L^{1} \cap L^{\infty} \times \mathbb{R}$ by a standard contractionmapping argument 1

\footnotetext{
${ }^{1}$ Specifically, for initial time $T \geq 0$, and $t \geq T$, split the expression (3.21) for $\dot{\alpha}(t)$ into the sum of a bounded "known" term $-\int_{-\infty}^{\infty} e_{t}(y, t) u_{0}(y) d y+\int_{0}^{T} \int_{-\infty}^{+\infty} e_{y t}(y, t-s)(N(u)+\dot{\alpha} u)(y, s) d y d s$ and an "unknown term" $\int_{T}^{t} \int_{-\infty}^{+\infty} e_{y t}(y, t-s)(N(u)+\dot{\alpha} u)(y, s) d y d s$ that is contractive for $(u, \dot{\alpha})$ bounded and $|t-T| \ll 1$. The $u$-equation (3.2) may be treated in standard fashion, treating the right-hand side as a forcing term and expressing $u$ as an integral on $[T, t]$, again contractive for $|t-T| \ll 1$.
} 
REMARK 3.5. Here, the key step in deriving (3.20) is to observe that the contribution in the right-hand side of (3.18) coming from terms involving $\bar{u}^{\prime}(x) e(y, t)$ is, under definition (3.19), exactly $-\bar{u}^{\prime}(x) \alpha(t)$, so cancels the final term. That is, we have defined the instantaneous translation $\alpha(t)$ from considerations of technical convenience so as to cancel all nondecaying terms in (3.18). Note that $\alpha(t)$ agrees to linear order with the prescription $\sigma(t)$ in Remark 3.2 of the instantaneous shock shift for the linearized equations.

3.3. Nonlinear iteration. Associated with the solution $(u, \dot{\alpha})$ of the integral system (3.20)-(3.21), define

$$
\zeta(t):=\sup _{0 \leq s \leq t, 1 \leq p \leq \infty}\left(|u|_{L^{p}}(s)(1+s)^{\frac{1}{2}(1-1 / p)}+|\dot{\alpha}(s)|(1+s)^{1 / 2}\right) .
$$

Lemma 3.6. For all $t \geq 0$ for which $\zeta(t)$ is finite, some $C>0$, and $E_{0}:=\left|u_{0}\right|_{L^{1} \cap L^{\infty}}$,

$$
\zeta(t) \leq C\left(E_{0}+\zeta(t)^{2}\right)
$$

Proof. With the established bounds on $\tilde{G}$ and $e$, the proof of (3.23) is almost identical to that of (2.13) in the constant-coefficient case. Noting, by quadratic dependence, $N(u)=O\left(|u|^{2}\right)$ and the definition (2.12) of $\zeta$, that

$$
\begin{aligned}
& |N(u)+| \dot{\alpha}|u|_{L^{1}} \leq C|u|_{L^{1}}\left(|u|_{L^{\infty}}+|\alpha|\right) \leq \zeta(t)^{2}(1+t)^{-\frac{1}{2}} \\
& |N(u)+| \dot{\alpha}|u|_{L^{p}} \leq C|u|_{L^{p}}\left(|u|_{L^{\infty}}+|\alpha|\right) \leq \zeta(t)^{2}(1+t)^{-\frac{1}{2}(1-1 / p)-\frac{1}{2}},
\end{aligned}
$$

we obtain, similarly as in (2.15), applying Lemmas 3.13 3.14 to representation (3.20),

$$
\begin{aligned}
|u(\cdot, t)|_{L^{p}(x)} \leq & C(1+t)^{-\frac{1}{2}(1-1 / p)} E_{0}+C \zeta(t)^{2} \int_{0}^{t / 2}(t-s)^{-\frac{1}{2}(1-1 / p)-1 / 2}(1+s)^{-\frac{1}{2}} d s \\
& +C \zeta(t)^{2} \int_{t / 2}^{t}(t-s)^{-\frac{1}{2}}(1+s)^{-\frac{1}{2}(1-1 / p)-\frac{1}{2}} d s \\
\leq & C\left(E_{0}+\zeta(t)^{2}\right)(1+t)^{-\frac{1}{2}(1-1 / p)} .
\end{aligned}
$$

Similarly, by (3.15) and (3.16),

$$
\begin{aligned}
|\dot{\alpha}(t)| \leq & C(1+t)^{-\frac{1}{2}} E_{0}+C \zeta(t)^{2} \int_{0}^{t / 2}(t-s)^{-1}(1+s)^{-\frac{1}{2}} d s \\
& +C \zeta(t)^{2} \int_{t / 2}^{t}(t-s)^{-\frac{1}{2}}(1+s)^{-1} d s \\
\leq & C\left(E_{0}+\zeta(t)^{2}\right)(1+t)^{-\frac{1}{2}} .
\end{aligned}
$$

Combining and rearranging (3.25) $-(3.28)$, we obtain (2.3).

Corollary 3.7 (Stability of shock solutions). Viscous shock solutions $\bar{u}(x)$ of (1.1) are nonlinearly stable in $L^{1} \cap L^{\infty}$ and nonlinearly orbitally asympotically stable in $L^{p}$, $p>1$, with respect to initial perturbations $u_{0}$ that are sufficiently small in $L^{1} \cap L^{\infty}$. 
More precisely, for some $C>0$ and $\alpha \in W^{1, \infty}(t)$,

$$
\begin{aligned}
|\tilde{u}-\bar{u}(\cdot-\alpha)|_{L^{p}}(t) & \leq\left. C(1+t)^{-\frac{1}{2}(1-1 / p)}|\tilde{u}-\bar{u}|_{L^{1} \cap L^{\infty}}\right|_{t=0}, \\
|\dot{\alpha}(t)| & \leq\left. C(1+t)^{-\frac{1}{2}}|\tilde{u}-\bar{u}|_{L^{1} \cap L^{\infty}}\right|_{t=0}, \\
|\alpha(t)| & \leq\left. C|\tilde{u}-\bar{u}|_{L^{1} \cap L^{\infty}}\right|_{t=0}, \\
|\tilde{u}-\bar{u}|_{L^{1} \cap L^{\infty}}(t) & \leq\left. C|\tilde{u}-\bar{u}|_{L^{1} \cap L^{\infty}}\right|_{t=0},
\end{aligned}
$$

for all $t \geq 0,1 \leq p \leq \infty$, for solutions $\tilde{u}$ of (1.1) with $\left.|\tilde{u}-\bar{u}|_{L^{1} \cap L^{\infty}}\right|_{t=0}$ sufficiently small.

Proof. The first two inequalities follow by a proof identical to that of Proposition 2.4 in the constant-coefficient case, using (3.6) and continuity of $\zeta$ wherever $\zeta$ is finite, a consequence of short-time existence theory, to obtain $\zeta(t) \leq 2 C E_{0}$, for $E_{0}:=$ $\left.|\tilde{u}-\bar{u}|_{L^{1} \cap L^{\infty}}\right|_{t=0} \leq \eta_{0}$ sufficiently small. This yields the first two bounds by definition of $\zeta$. The third then follows using (3.17), by

$$
\begin{aligned}
|\alpha(t)| \leq & C E_{0}+C \zeta(t)^{2} \int_{0}^{t / 2}(t-s)^{-\frac{1}{2}}(1+s)^{-\frac{1}{2}} d s \\
& +C \zeta(t)^{2} \int_{t / 2}^{t}(t-s)^{-\frac{1}{2}}(1+s)^{-\frac{1}{2}} d s \\
\leq & C\left(E_{0}+\zeta(t)^{2}\right) .
\end{aligned}
$$

Finally, we note that

$$
\tilde{u}(x, t)-\bar{u}(x)=u(x-\alpha(t), t)-(\bar{u}(x)-\bar{u}(x-\alpha(t)),
$$

so that $|\tilde{u}(\cdot, t)-\bar{u}|$ is controlled by the sum of $|u|$ and $|\bar{u}(x)-\bar{u}(x-\alpha(t))| \sim \alpha(t)\left|\bar{u}^{\prime}(x)\right|$, hence, by our estimates, remains $\leq C E_{0}$ for all $t \geq 0$, for $E_{0}$ sufficiently small. This verifies the fourth inequality, yielding nonlinear stability and completing the result.

REMARK 3.8. In the semilinear case considered here, Corollary 3.7 could be proved in a more straightforward fashion by a contraction-mapping argument applied directly to the system (3.20)-(3.21), bypassing the continuous induction argument above. However, in more delicate situations such as the quasilinear parabolic or hyperbolic-parabolic cases, it is advantageous for reasons of regularity to separate the issues of short-time existence/well-posedness and long-time bounds, as we have done here; see MaZ2, MaZ4, Z4, RZ] for further discussion.

REMARK 3.9. Again, the rate of decay (3.27) (i) is that of a heat kernel; that is, the mechanism for stability is diffusive only, and not involving compressivity of the shock. This rate is in fact sharp, as may be seen intuitively by considering a compactly supported, perturbation supported, arbitrarily far from the shock location $x=0$. Far from the shock, the background solution $\bar{u}$ is approximately constant, and so behavior is like that of a perturbation of a constant solution as studied in Section 2. But, this is readily seen to decay like a heat kernel, giving the stated rate (3.27)(i).

3.4. Postscript: phase-asymptotic vs. asymptotic orbital stability. A stronger condition than nonlinear orbital stability, proved above, is nonlinear phase-asymptotic orbital stability, in which a perturbed solution $\tilde{u}$ is required to approach not only the set of translates of $\bar{u}$, but a specific translate of $u$. In the language of Corollary 3.7, this amounts to the requirement that $\alpha$ have a limit $\alpha(t) \rightarrow \alpha(+\infty)$ as $t \rightarrow+\infty$. 
We do not establish this property in Corollary 3.7 nor is it established in [Z1, MaZ2, MaZ4. Indeed, for the general class of perturbations considered here (and in Z1, MaZ2, MaZ4]), $\alpha(t)$ if it converges to a limit does not do so at any uniform algebraic rate depending only on $E_{0}, t$, as may be seen by considering perturbations with support arbitrarily far from the shock location $x=0$. See [Z1] for further discussion.

It is a strength of this approach that such data may be treated nonetheless, and in a simple fashion parallel to the treatment of the constant-coefficient case. However, phaseasymptotic stability does not seem to be accessible by this simple argument scheme involving only $L^{p} \rightarrow L^{q}$ estimates. For proofs of phase-asymptotic stability under strengthened assumptions on the initial data, following a similar tracking approach but involving additional pointwise information on the solution, see [R, HZ, HR, HRZ, RZ].

4. The system case. We have described the nonlinear stability argument of Z1, MaZ2, MaZ4 in the simple scalar setting of Burgers equation. We now discuss briefly how this carries over to the case of general hyperbolic-parabolic systems, including NavierStokes equations of compressible gas dynamics and MHD. Namely, Remark 3.4 plus essentially the same argument described here gives nonlinear orbital stability of viscous shocks provided that they satisfy an Evans function (generalized spectral stability) assumption yielding the necessary pointwise bounds. The Evans condition is necessary for linearized stability as shown in [ZH, MaZ3. It holds always for small-amplitude shocks, but may fail in general for large-amplitude shocks. In the large-amplitude case, it is readily checked numerically; in certain special limits, it may be checked analytically using asymptotic ODE and/or singular perturbation theory. When the Evans condition fails, there are interesting implications for dynamics/bifurcation; see [Z5, Z7, TZ1, TZ2, TZ3, TZ4, SS, BeSZ].

See [AGJ, GZ, ZH, ZS, MaZ3 for a discussion of the Evans function and its origins. For verification of the Evans condition for small-amplitude shocks, see [ZH, HuZ, PZ, FS1]. For examples of unstable shocks, see [GZ, ZS. For numerical and analytical verification for large-amplitude shocks, see [BHZ, BHRZ, [HLZ, HLyZ, CHNZ; see [Br, BrZ, BDG, HuZ2] for a more general discussion of numerical Evans function techniques. See [ZS, Z2, Z4, Z6, GMWZ, GMWZ2, [FS2] for extensions to multiple dimensions.

The derivation of pointwise Green function bounds for general systems is complicated, involving detailed estimates on the resolvent kernel using the Evans function and asymptotic ODE techniques, converted to bounds on the Green kernel via stationary phase estimates in the inverse Laplace transform formula. See [ZH, Z3, Z2, Z4, BeSZ, GMWZ, GMWZ2 for discussions of these and related techniques. These are details of the linear theory. Here, we have chosen to isolate the nonlinear iteration argument by restricting to a case (Burgers equation) for which the linear theory is explicitly known a priori, in order to give the reader a flavor of the arguments.

We emphasize: once the linearized theory is established, the nonlinear shock-tracking argument of [Z1, MaZ2, MaZ4, Z2] is essentially the same for the system case as for the scalar case. See Remark 3.4. Indeed, the method is of a still more general nature; see the parallel treatments of stability of relaxation shocks and detonation waves in 
MaZ1, LRTZ, TZ4 and of spatially periodic solutions of viscous conservation laws or balance laws in [JZ1, JZ2, JZN].

Acknowledgement. Thanks go to Mark Williams and Benjamin Texier for their interest in the work, and for several helpful comments improving the exposition.

\section{Appendices.}

Appendix A. Comparison with previous work. The instantaneous tracking method used here is to be contrasted with an earlier method introduced in Liu85, Liu97] and completed in SXX, LZ2, 2 based on a fixed time-asymptotic shock location determined by conservation of mass. There, the approach is, rather than defining an instantaneous shock location so as to minimize residual error, to develop a detailed approximate solution sufficiently accurate that the nonlinear residual is again small enough to close a nonlinear iteration. This has the advantage that, if one can succeed, one automatically obtains additional details of the solution. It has the disadvantage that one must obtain such details in order for the argument to close, and this means a rather complicated and technical argument, and one that is difficult to carry out or check. Moreover, as described in Section 3.4, such methods require localization of the initial perturbation in the form of algebraic spatial decay rates. The recent paper [LZ2, on the other hand, features a uniform basin of attraction as the shock amplitude goes to zero, something that is not attained from the basic stability argument given in Section 3 we discuss this issue further in Appendix B] Note that the results of [SX, LZ2 apply only to smallamplitude "Lax type" waves and artificial (Laplacian) viscosity, whereas our results in [Z1, MaZ4, Z4, HZ, RZ] apply in principle to waves of arbitrary amplitude and type and physical (partially parabolic) viscosity; see [HLZ, BHZ for proofs of stability in the large-amplitude limit for isentropic gas dynamics and MHD.

It should be mentioned that the early paper [LZu involving shock tracking of undercompressive shocks is based on time-asymptotic estimation of the shock location, in this case obtained by a time-asymptotic study of the linearized equations and not simply conservation of mass, rather than instantaneous tracking in the sense of [Z1, MaZ2] described above. This does not lead to a fixed estimate of shock location, due to interaction with nonlinear terms, but is less accurate than the instantaneous scheme used in [HZ].

${ }^{2}$ Both [Liu85] and Liu97] contain significant errors, corrected respectively in [SX] and [LZ2]. A key step in Liu85 is the intermediate result, stated in the final paragraph of p. 12, that

$$
\int_{0}^{+\infty} \int_{-\infty}^{+\infty}|u(x, t)-\bar{u}(x-\delta)-\Theta(x, t)|^{2} d x d t<\infty
$$

where $\Theta$ is defined as a sum of "outgoing diffusion waves"; as shown by an explicit counterexample in [GSZ], this estimate in general does not hold. Likewise, it is pointed out in [LZ2] that [Liu97] contains an "incorrect Ansatz" $(|x|+t+1)^{-1 / 2}(1+|x|)^{-1}$ of the perturbation near the shock location (centered at $x=0$ in the analysis), which is described in [LZ2 as algebraically decaying, hence not sufficiently confined to the shock layer for the argument of Liu97 to close. Both of these errors are related to the contribution $\bar{u}(x-\delta(t))-\bar{u}(x) \sim \delta(t) \bar{u}^{\prime}(x)$ removed in our argument by instantaneous shock tracking. As pointed out in Remark 2, [HZ], this contribution corresponds in turn to the "coupled diffusion wave" introduced by different means in $[\mathrm{SX}]$ in order to correct the argument of Liu85]. 
It can succeed in $[\mathrm{LZu}]$ because of the advantageous structure of the model considered, but does not appear sufficient in the general case considered in [HZ]. Similarly, the early shock-tracking scheme used in [LY] is based on conservation of mass and not a more detailed localized fit as in the instantaneous tracking described above; this suffices to treat the scalar problem considered there, but does not appear sufficiently accurate to treat the system case.

Appendix B. The small-amplitude limit. It is instructive to consider the smallamplitude limit $\left|u_{+}-u_{-}\right| \rightarrow 0$. Consider now the family of stationary viscous shock solutions

$$
\bar{u}^{\varepsilon}(x):=-\varepsilon \tanh (\varepsilon x / 2), \quad \lim _{x \rightarrow \pm \infty} \bar{u}^{\varepsilon}(x)=\bar{u}_{ \pm}^{\varepsilon}=\mp \varepsilon
$$

of (1.1), and examine the behavior as $\varepsilon \rightarrow 0$.

Denote the associated homogeneous linearized equation by

$$
v_{t}-L^{\varepsilon} v=v_{t}+\left(a^{\varepsilon}(x) v\right)_{x}-v_{x x}=0,\left.\quad v\right|_{t=0}=f,
$$

where $a^{\varepsilon}(x):=\bar{u}^{\varepsilon}(x)$. The invariance $(x, t, u) \rightarrow\left(x / \varepsilon, t / \varepsilon^{2}, u / \varepsilon\right)$ of Burgers equation converts this to the $\varepsilon$-independent case (1.5) considered in Section 3 , from which we may deduce the $\varepsilon$-dependent Green function formula

$$
e^{L^{\varepsilon} t} f=\int_{-\infty}^{+\infty} G^{\varepsilon}(x, t ; y) f(y) d y,
$$

where

$$
\begin{aligned}
& G^{\varepsilon}(x, t ; y):=e^{L^{\varepsilon} t} \delta_{y}(x)=\left(\bar{u}^{\varepsilon}\right)^{\prime}(x)\left(\frac{1}{2 \varepsilon}\right)\left(\operatorname{errfn}\left(\frac{x-y-\varepsilon t}{\sqrt{4 t}}\right)-\operatorname{errfn}\left(\frac{x-y+\varepsilon t}{\sqrt{4 t}}\right)\right) \\
& +\left(\left(\frac{e^{-\frac{\varepsilon x}{2}}}{e^{\frac{\varepsilon x}{2}}+e^{-\frac{\varepsilon x}{2}}}\right) \frac{e^{-\frac{(x-y-\varepsilon t)^{2}}{4 t}}}{\sqrt{4 \pi t}}+\left(\frac{e^{\frac{\varepsilon x}{2}}}{e^{\frac{\varepsilon x}{2}}+e^{-\frac{\varepsilon x}{2}}}\right) \frac{e^{-\frac{(x-y+\varepsilon t)^{2}}{4 t}}}{\sqrt{4 \pi t}}\right)
\end{aligned}
$$

and $\left(\bar{u}^{\varepsilon}\right)^{\prime}(x)=\varepsilon^{2} \bar{u}^{\prime}(\varepsilon x) \sim \varepsilon^{2} e^{-\theta \varepsilon|x|}, \theta>0$. Here, we are using the scaling relations

$$
\bar{u}^{\varepsilon}(x)=\varepsilon \bar{u}(\varepsilon x) \quad \text { and } \quad G^{\varepsilon}(x, t ; y)=\varepsilon G\left(\varepsilon x, \varepsilon^{2} t ; \varepsilon y\right) .
$$

Decompose again

$$
G^{\varepsilon}(x, t ; y):=E^{\varepsilon}(x, t ; y)+S^{\varepsilon}(x, t ; y)+R^{\varepsilon}(x, t ; y),
$$

where

$$
\begin{gathered}
E^{\varepsilon}(x, t ; y):=\left(\bar{u}^{\varepsilon}\right)^{\prime}(x) e^{\varepsilon}(y, t), \quad e^{\varepsilon}(y, t):=\left(\frac{1}{2 \varepsilon}\right)\left(\operatorname{errfn}\left(\frac{-y-\varepsilon t}{\sqrt{4 t}}\right)-\operatorname{errfn}\left(\frac{-y+\varepsilon t}{\sqrt{4 t}}\right)\right), \\
S^{\varepsilon}(x, t ; y):=\left(\left(\frac{e^{-\frac{\varepsilon x}{2}}}{e^{\frac{\varepsilon x}{2}}+e^{-\frac{\varepsilon x}{2}}}\right) \frac{e^{-\frac{(x-y-\varepsilon t)^{2}}{4 t}}}{\sqrt{4 \pi t}}+\left(\frac{e^{\frac{\varepsilon x}{2}}}{e^{\frac{\varepsilon x}{2}}+e^{-\frac{\varepsilon x}{2}}}\right) \frac{e^{-\frac{(x-y+\varepsilon t)^{2}}{4 t}}}{\sqrt{4 \pi t}}\right),
\end{gathered}
$$

and

$$
\begin{aligned}
R^{\varepsilon}(x, t ; y):= & \left(\bar{u}^{\varepsilon}\right)^{\prime}(x)\left(\frac{1}{2 \varepsilon}\right)\left(\operatorname{errfn}\left(\frac{x-y-\varepsilon t}{\sqrt{4 t}}\right)-\operatorname{errfn}\left(\frac{-y-\varepsilon t}{\sqrt{4 t}}\right)\right) \\
& +\left(\bar{u}^{\varepsilon}\right)^{\prime}(x)\left(\frac{1}{2 \varepsilon}\right)\left(\operatorname{errfn}\left(\frac{-y+\varepsilon t}{\sqrt{4 t}}\right)-\operatorname{errfn}\left(\frac{x-y+\varepsilon t}{\sqrt{4 t}}\right)\right) .
\end{aligned}
$$


Defining the perturbation

$$
u(x, t):=\tilde{u}(x+\alpha(t), t)-\bar{u}^{\varepsilon}(x),
$$

setting $\tilde{G}^{\varepsilon}:=S^{\varepsilon}+R^{\varepsilon}$, and following the steps of Section 3, we obtain again the integral representation

$$
\begin{aligned}
u(x, t)= & \int_{-\infty}^{\infty} \tilde{G}^{\varepsilon}(x, t ; y) u_{0}(y) d y \\
& -\int_{0}^{t} \int_{-\infty}^{\infty} \tilde{G}_{y}^{\varepsilon}(x, t-s ; y)(N(u)+\dot{\alpha} u)(y, s) d y d s, \\
\dot{\alpha}(t)=- & \int_{-\infty}^{\infty} e_{t}^{\varepsilon}(y, t) u_{0}(y) d y \\
& +\int_{0}^{t} \int_{-\infty}^{+\infty} e_{y t}^{\varepsilon}(y, t-s)(N(u)+\dot{\alpha} u)(y, s) d y d s . \\
\alpha(t)=- & \int_{-\infty}^{\infty} e^{\varepsilon}(y, t) u_{0}(y) d y \\
& +\int_{0}^{t} \int_{-\infty}^{+\infty} e_{y}^{\varepsilon}(y, t-s)(N(u)+\dot{\alpha} u)(y, s) d y d s .
\end{aligned}
$$

Dependence on $\varepsilon$. Evidently, we could carry through the entire stability analysis of Section 3, as the $\varepsilon$-dependent Green function $G^{\varepsilon}=E^{\varepsilon}+S^{\varepsilon}+R^{\varepsilon}$ has the same form as $G$. However, the bounds obtained in this way, in particular, the estimate (3.11) on the remainder $R$, would involve constants $C=C(\varepsilon)>0$ blowing up as $\varepsilon \rightarrow 0$. This means that the allowable size $E_{0} \leq \frac{1}{4 C(\varepsilon)^{2}}$ of perturbations, determined in the proof of Corollary 2.4. goes to zero as $\varepsilon \rightarrow 0$. That is, the basin of attraction of the shock $\bar{u}^{\varepsilon}$ established by our basic stability argument shrinks to zero as $\varepsilon \rightarrow 0$. Indeed, the bounds derived for general systems in [ZH, MaZ3] (described briefly in Section 4) share this same property, and so the basin of attraction for the stability results proved in [MaZ2, MaZ4, Z1, Z2, HZ] and related works goes to zero as the shock amplitude goes to zero.

However, this is not an inherent limitation of the method, or the shock. Following, we show that by different, more careful, estimates of $E^{\varepsilon}$ and $R^{\varepsilon}$, we may in fact recover a uniform stability result, valid for perturbations of sufficiently small size independent of $\varepsilon$.

Proposition B.1. For some $C>0$ independent of $\varepsilon, 0<\varepsilon \leq 1$, and all $t>0$,

$$
\begin{gathered}
\left|\int_{-\infty}^{+\infty} \tilde{G}^{\varepsilon}(x, t ; y) f(y) d y\right|_{L^{p}(x)} \leq C t^{-\frac{1}{2}(1-1 / p)}|f|_{L^{1}} \\
\left|\int_{-\infty}^{+\infty} \tilde{G}_{y}^{\varepsilon}(x, t ; y) f(y) d y\right|_{L^{p}(x)} \leq C t^{-\frac{1}{2}(1-1 / p)-\frac{1}{2}}|f|_{L^{1}} \\
\left|\int_{-\infty}^{+\infty} \tilde{G}^{\varepsilon}(x, t ; y) f(y) d y\right|_{L^{p}(x)} \leq C|f|_{L^{p}}, \quad\left|\int_{-\infty}^{+\infty} \tilde{G}_{y}^{\varepsilon}(x, t ; y) f(y) d y\right|_{L^{p}(x)} \leq C t^{-\frac{1}{2}}|f|_{L^{p}} ; \\
\left|\int_{-\infty}^{+\infty} e_{t}^{\varepsilon}(y, t) f(y) d y\right| \leq C t^{-\frac{1}{2}}|f|_{L^{1}}, \quad\left|\int_{-\infty}^{+\infty} e_{t y}^{\varepsilon}(y, t) f(y) d y\right| \leq C t^{-1}|f|_{L^{1}}
\end{gathered}
$$




$$
\left|\int_{-\infty}^{+\infty} e_{t}^{\varepsilon}(x, t ; y) f(y) d y\right| \leq C|f|_{L^{\infty}} ; \quad\left|\int_{-\infty}^{+\infty} e_{y t}^{\varepsilon}(x, t ; y) f(y) d y\right| \leq C t^{-\frac{1}{2}}|f|_{L^{\infty}}
$$

and

$$
\left|\int_{-\infty}^{+\infty} e^{\varepsilon}(y, t) f(y) d y\right| \leq C \varepsilon^{-1}|f|_{L^{1}}, \quad\left|\int_{-\infty}^{+\infty} e_{y}^{\varepsilon}(y, t) f(y) d y\right| \leq C \varepsilon^{-1} t^{-1 / 2}|f|_{L^{1}} .
$$

Proof. As $S^{\varepsilon}$ evidently obeys the same decay estimates as $S$, to establish the stated bounds on $\tilde{G}^{\varepsilon}$, it is sufficient to establish them for $R^{\varepsilon}$. This is a straightforward consequence of Lemmas D.4 and D.5 established in Appendix D.3. Likewise, for the stated bounds on $e^{\varepsilon}$, it is sufficient to establish corresponding $L^{p}(y)$ bounds on $\varepsilon^{\varepsilon}$, from which the results then follow by Hölder's inequality. The needed bounds are established in Lemma D.3. Appendix D.2.

Corollary B.2 (Stability of small-amplitude shock solutions). For $0<\varepsilon \leq 1$, viscous shock solutions $\bar{u}^{\varepsilon}(x)$ of (1.1) are nonlinearly stable in $L^{1} \cap L^{\infty}$ and nonlinearly orbitally asympotically stable in $L^{p}, p>1$, with respect to initial perturbations $u_{0}$ with $L^{1} \cap L^{\infty}$ norm less than or equal to $\eta_{0}>0$ sufficiently small, where $\eta_{0}$ is independent of $0<\varepsilon \leq 1$. More precisely, for some $C>0$ independent of $0<\varepsilon \leq 1$, there is $\alpha \in W^{1, \infty}(t)$ such that

$$
\begin{aligned}
\left|\tilde{u}-\bar{u}^{\varepsilon}(\cdot-\alpha)\right|_{L^{p}}(t) & \leq C(1+t)^{-\frac{1}{2}(1-1 / p)} E_{0}, \\
|\dot{\alpha}(t)| & \leq C(1+t)^{-\frac{1}{2}} E_{0}, \\
|\alpha(t)| & \leq C \varepsilon^{-1} E_{0}, \\
\left|\tilde{u}-\bar{u}^{\varepsilon}\right|_{L^{1} \cap L^{\infty}}(t) & \leq C E_{0},
\end{aligned}
$$

for all $t \geq 0,1 \leq p \leq \infty$, for solutions $\tilde{u}$ of (1.1) with $E_{0}:=\left.\left|\tilde{u}-\bar{u}^{\varepsilon}\right|_{L^{1} \cap L^{\infty}}\right|_{t=0} \leq \eta_{0}$.

Proof. The proof of the first two bounds follows exactly as in the proof of Corollary 3.7 in the fixed-amplitude case, since the integral equations for $(u, \dot{\alpha})$ form a closed system involving only $\tilde{G}^{\varepsilon}, e_{t}^{\varepsilon}$ and $e_{y t}^{\varepsilon}$, and the bounds on $\tilde{G}^{\varepsilon}, e_{t}^{\varepsilon}$ and $e_{y t}^{\varepsilon}$ are the same as the bounds on $\tilde{G}, e_{t}$ and $e_{y t}$ in the fixed-amplitude case. With these bounds established, we obtain the third bound from (B.12), using the fact that the bounds on $e^{\varepsilon}$ and $e_{y}^{\varepsilon}$ are no worse than $\varepsilon^{-1}$ times the bounds on $e$ and $e_{y}$ in the fixed-amplitude case.

Finally, we note that $\tilde{u}(x, t)-\bar{u}^{\varepsilon}(x)=u(x-\alpha(t), t)-\left(\bar{u}^{\varepsilon}(x)-\bar{u}^{\varepsilon}(x-\alpha(t))\right.$, so that $\left|\tilde{u}(\cdot, t)-\bar{u}^{\varepsilon}\right|$ is controlled by the sum of $|u|$ and $\left|\bar{u}^{\varepsilon}(x)-\bar{u}^{\varepsilon}(x-\alpha(t))\right|$. By monotonicity of scalar shock profiles as orbits of the first-order scalar profile ODE (1.4), $\bar{u}^{\varepsilon}(x)-\bar{u}^{\varepsilon}(x-$ $\alpha(t))$ has one sign, hence

$$
\left|\bar{u}^{\varepsilon}(x)-\bar{u}^{\varepsilon}(x-\alpha(t))\right|_{L^{1}}=\left|\int_{-\infty}^{+\infty}\left(\bar{u}^{\varepsilon}(x)-\bar{u}^{\varepsilon}(x-\alpha(t))\right) d x\right|=|\alpha(t)|\left|u_{+}^{\varepsilon}-u_{-}^{\varepsilon}\right|,
$$

and, by (B.18) (iii),

$$
\left|\bar{u}^{\varepsilon}(x)-\bar{u}^{\varepsilon}(x-\alpha(t))\right|_{L^{1}}=2 \varepsilon|\alpha(t)| \leq 2 C E_{0} .
$$

Likewise, by the Mean Value Theorem,

$$
\left|\bar{u}^{\varepsilon}(x)-\bar{u}^{\varepsilon}(x-\alpha(t))\right| \leq\left.|\alpha(t)|\left(\bar{u}^{\varepsilon}\right)^{\prime}\right|_{L^{\infty}} \leq\left(C E_{0} / \varepsilon\right)\left(\varepsilon^{2}\right)=C E_{0} \varepsilon,
$$


by the asymptotics $\left(\bar{u}^{\varepsilon}\right)^{\prime} \sim \varepsilon^{2} e^{-\theta \varepsilon|x|}$. Thus, $\left|\bar{u}^{\varepsilon}(x)-\bar{u}^{\varepsilon}(x-\alpha(t))\right|_{L^{1} \cap L^{\infty}} \leq C E_{0}$, and so $\left|\tilde{u}(x, t)-\bar{u}^{\varepsilon}(x)\right|_{L^{1} \cap L^{\infty}} \leq C E_{0}$ for all $t \geq 0$, for $E_{0}$ sufficiently small. This verifies the fourth inequality, yielding nonlinear stability and completing the result.

REmark B.3. In the small-amplitude limit $\varepsilon \rightarrow 0$, the shock shift $\alpha \rightarrow+\infty$ as $\varepsilon^{-1}$ times perturbation mass. Nonetheless, the stability estimates are uniform, independent of $\varepsilon$.

Remark B.4. As discussed in Section 3.4, we have obtained stability for a class $L^{1} \cap L^{\infty}$ of perturbations that lead to shock shifts $\alpha$ not only of order $1 / \varepsilon$, but also decaying subalgebraically to their limits $\alpha(+\infty)$, if they exist.

REMARK B.5. Here we have treated only the simple and explicit case of Burgers equation. It would be very interesting to try to treat the small-amplitude system case by a similarly simple argument based on this approach, using the singular perturbation techniques developed in $\mathrm{MaZ3}, \mathrm{PZ}$ to obtain the necessary sharpened $\varepsilon$-dependent bounds analogous to those of Proposition B.1 in the Burgers case to try to obtain results uniform in $\varepsilon$.

Appendix C. Alternative shock-tracking schemes. As discussed in Remarks 3.2 and 3.4, the quantity $\alpha(t)$, introduced for technical reasons in (3.19), has an interpretation as an "instantaneous shock shift", measuring the approximate location of a perturbed viscous shock profile at time $t$. This suggests the question what is the "exact" location of an asymptotic shock profile, and how well $\alpha(t)$ approximates this location. The study of this question leads to an interesting class of alternative shock-tracking schemes that are time-asymptotically equivalent to (3.20)-(3.21), based on localized projections, converging in the "infinite-localization" limit to a pointwise phase condition introduced in GMWZ] in the context of the small-viscosity limit.

Unlike a perturbed inviscid shock wave, which is sharply located by the presence of a discontinuity, a perturbed viscous shock wave is smooth, so requires some extrinsic criterion to define its location. Two intuitive definitions immediately come to mind. The first, defining the location of an unperturbed stationary scalar shock $u \equiv \bar{u}(x)$ without loss of generality to be the origin, $x=0$, is simply to define the location $\alpha(t)$ of a perturbed shock $\tilde{u}$ as the point $\alpha(t)$ at which $\tilde{u}$ takes on the value $\bar{u}(0)$, or

$$
\tilde{u}(\alpha(t), t)=\bar{u}(0) .
$$

By the Implicit Function Theorem and the fact that $\bar{u}^{\prime}(0) \neq 0$ (recall that $\bar{u}$ is monotone, as the solution of a scalar first-order traveling-wave ODE), this uniquely defines $\alpha$ for $\left|\tilde{u}^{\prime}-\bar{u}^{\prime}\right|_{L^{\infty}}(t)$ sufficiently small.

In the system case $u \in \mathbb{R}^{n}$, we cannot satisfy (C.1) for all $n$ coordinates using the single parameter $\alpha$, so we must choose some preferred coordinate direction, substituting for (C.1) the system analog

$$
\ell \cdot \tilde{u}(\alpha(t), t)=\ell \cdot \bar{u}(0)
$$

for some vector $\ell \in \mathbb{R}^{n}$ such that $\ell \cdot \bar{u}^{\prime}(0) \neq 0$, a condition that, by the Implicit Function Theorem, guarantees that $\alpha(t)$ is well defined for $\left|\tilde{u}^{\prime}-\bar{u}^{\prime}\right|_{L^{\infty}}(t)$ sufficiently small.

Defining the perturbation variable

$$
u(x, t)=\tilde{u}(x+\alpha(t), t)-\bar{u}(x),
$$


following the notation of Section 3, we find that (C.2) translates to the phase condition

$$
\ell \cdot u(0, t)=0,
$$

determining $\alpha(t)$ implicitly through (C.3). Condition (C.4) is particularly natural from the point of view of the resolvent equation arising in the solution by Laplace transform of the associated linearized equations. For, the resolvent equation consists of an underdetermined ordinary differential boundary-value problem for which the standard treatment is to remove indeterminacy by one or more phase conditions such as (C.4). Indeed, this condition was introduced in [GMWZ starting from just such considerations, for the study of shock stability in the vanishing viscosity limit 3

The second intuitive definition is, following Goodman [G], to define the shock shift $\alpha$ so as to minimize the least squares distance of $\tilde{u}(x, t)$ from the shifted shock $\bar{u}(x-\alpha(t))$, that is, to minimize $|u(\cdot, t)|_{L^{2}}$. This leads to the "localized projection condition" (EulerLagrange equation)

$$
\langle\ell, u\rangle_{L^{2}}=0, \quad\left\langle\ell, \bar{u}^{\prime}\right\rangle_{L^{2}}=1
$$

where $\ell(x):=\frac{\bar{u}^{\prime}(x)}{\left|\bar{u}^{\prime}\right|_{L^{2}}^{2}}$ (see Appendix D.4 for this calculation). Here, the word "localized" refers to the fact that $\ell(x)$ decays as $x \rightarrow \pm \infty$. More generally, we denote as a localized projective condition any condition of the form (C.5) with $\ell \in L^{1}$. This can be viewed as a nonlocal version of the pointwise phase condition (C.4), converging to (C.4) in the "infinite-localization limit" $\ell(x) \rightarrow \ell_{0} \delta(x), \ell_{0} \in \mathbb{R}^{n}$ constant, of a Dirac measure.

Each of these schemes (either of the form (C.4) or (C.5)) may be written as an evolution equation in $(u, \alpha)$. Defining the perturbation variable $u$ of (C.3), we find as in Section 3 that $u$ obeys the partial differential equation

$$
u_{t}-L u=N(u)_{x}+\dot{\alpha}\left(\bar{u}_{x}+u_{x}\right)
$$

depending on $\dot{\alpha}$, defined implicitly by (C.5). Differentiating (C.5) with respect to $t$, we obtain

$$
0=\left\langle\ell, u_{t}\right\rangle_{L^{2}}=\left\langle\ell, L u+N(u)_{x}+\dot{\alpha}\left(\bar{u}_{x}+u_{x}\right)\right\rangle_{L^{2}},
$$

which, using $\left\langle\ell, \bar{u}_{x}\right\rangle_{L^{2}}=1$, reduces to $\dot{\alpha}\left(1+\left\langle\ell, u_{x}\right\rangle_{L^{2}}\right)=-\left\langle\ell, L u+N(u)_{x}\right\rangle_{L^{2}}$, or, rearranging,

$$
\dot{\alpha}=-\frac{\left\langle\ell, L u+N(u)_{x}\right\rangle_{L^{2}}}{1+\left\langle\ell, u_{x}\right\rangle_{L^{2}}},
$$

well defined for $u \in H^{2}$ with $|u|_{H^{2}}$ sufficiently small. See [G, TZ1, Z7 for related discussions.

Together, (C.6)-C.7 determine a closed system of evolution equations for $(u, \dot{\alpha})$, similar in spirit to the system (3.20)-(3.21) of Section 3, but local in time, whereas the system (3.20)-3.21) involves "memory terms" depending on values of $u, \dot{\alpha}$ at earlier times $s \leq t$. For each choice of test function $\ell$, there results a different evolution system, and different solutions $(u, \dot{\alpha})$ and $\alpha$, representing different decompositions of the common solution $\tilde{u}$ of (1.1) under investigation, a perturbed viscous shock wave.

We know already from the analysis of Corollary 3.7 that the solution $\tilde{u}$ exists for all time and converges to the set of translates of the background shock $\bar{u}$. However, it is not

\footnotetext{
${ }^{3}$ More precisely, a multi-dimensional version reducing to C.4 in the one-dimensional case.
} 
a priori clear that the system (C.6) - C.7) has a global solution for any particular choice of $\ell$, nor that the solution $u$ should decay as $t \rightarrow 0$. That is, it is not clear which of these alternative shock-tracking schemes gives an accurate estimate of shock location in the sense that the known convergence of $\tilde{u}$ to the set of translates is revealed by decay at the appropriate rate of the perturbation variable $u$.

The following proposition asserts that all of these schemes are accurate in this sense, so that in principle any one of them could be used as the basis of an argument for nonlinear stability. Indeed, all lead to the same rates of decay.

Proposition C.1. Let $u^{\text {ref }}, \alpha^{\text {ref }}$ denote the solution of (3.20)-3.21) of Section 3, with initial data $\tilde{u}_{0}-\bar{u}, E_{0}:=\left|\tilde{u}_{0}-\bar{u}\right|_{L^{1} \cap H^{2}}$ sufficiently small, and let $u, \alpha$ denote the solution with the same initial data of (C.6)-(C.7), with $\ell \in L^{1}$. Then, $u, u^{\text {ref }}$ exist for all $t \geq 0$, with

$$
\begin{aligned}
|u|_{L^{p}}(t),\left|u^{\mathrm{ref}}\right|_{L^{p}}(t) & \leq C E_{0}(1+t)^{-\frac{1}{2}(1-1 / p),} \\
|u|_{H^{2}}(t),\left|u^{\mathrm{ref}}\right|_{H^{2}}(t) & \leq C E_{0}(1+t)^{-1 / 4}, \\
|u|_{L^{1} \cap H^{2}}(t)-\left|u^{\mathrm{ref}}\right|_{L^{1} \cap H^{2}}(t) & \leq C E_{0}(1+t)^{-1 / 2}, \\
|\tilde{u}-\bar{u}|_{L^{1} \cap H^{2}}(t) & \leq C E_{0}, \\
|\alpha|(t),\left|\alpha^{\mathrm{ref}}\right|(t) & \leq C E_{0}, \\
\left|\alpha-\alpha^{\mathrm{ref}}\right|(t) & \leq C E_{0}(1+t)^{-1 / 2} .
\end{aligned}
$$

Proof. A routine extension of the proof of Corollary 3.7, using the additional assumption of $H^{2}$ smallness of the initial data yields (3.27) augmented with $\left|u^{\text {ref }}\right|_{H^{2}}(t) \leq$ $C E_{0}(1+t)^{-\frac{1}{4}}$. We omit the details. (But see the results of [MaZ2, MaZ4] in the much more complicated system case.) The corresponding bounds (C.8)(i)-(ii), hence global existence of $u$, thus follow provided that we can establish (C.8)(iii).

Expanding

$$
\begin{aligned}
u(x, t) & =\tilde{u}(x+\alpha(t), t)-\bar{u}(x) \\
& =\tilde{u}(x+\alpha(t), t)-\bar{u}\left(x+\left(\alpha-\alpha^{\mathrm{ref}}\right)\right)+\bar{u}\left(x+\left(\alpha-\alpha^{\mathrm{ref}}\right)\right)-\bar{u}(x) \\
& =u^{\mathrm{ref}}\left(x+\left(\alpha-\alpha^{\mathrm{ref}}\right), t\right)+\left(\bar{u}\left(x+\left(\alpha-\alpha^{\mathrm{ref}}\right)\right)-\bar{u}(x)\right),
\end{aligned}
$$

we find using the triangle inequality, followed by the Mean Value Theorem together with exponential decay of $\bar{u}^{\prime}$, that

$$
|u|_{L^{1} \cap H^{2}}(t)-\left|u^{\mathrm{ref}}\right|_{L^{1} \cap H^{2}}(t) \leq\left|\bar{u}\left(x+\left(\alpha-\alpha^{\mathrm{ref}}\right)\right)-\bar{u}(x)\right|_{L^{1} \cap H^{2}} \leq C\left|\alpha-\alpha^{\mathrm{ref}}\right|(t),
$$

so that (C.8) (iii) follows from (C.8) (vi). Likewise, (iv) follows from (i)-(iii) and (v), which in turn follows from (vi) and the bounds on $\left|\alpha^{\text {ref }}\right|(t)$ established in Section 3 ,

Thus, it remains only to prove (C.8)(vi). Applying the definition $\langle\ell, u\rangle_{L^{2}}=0$ to expansion (C.9), we obtain

$$
\begin{aligned}
\left\langle\ell, u^{\mathrm{ref}}\left(x+\left(\alpha-\alpha^{\mathrm{ref}}\right), t\right)\right\rangle_{L^{2}} & =-\left\langle\ell, \bar{u}\left(x+\left(\alpha-\alpha^{\mathrm{ref}}\right)\right)-\bar{u}(x)\right\rangle_{L^{2}} \\
& =-\left\langle\ell,\left(\alpha-\alpha^{\mathrm{ref}}\right) \bar{u}^{\prime}+O\left(\left|\alpha-\alpha^{\mathrm{ref}}\right|^{2}\right)\right\rangle_{L^{2}} .
\end{aligned}
$$


Now applying $\left\langle\ell, \bar{u}^{\prime}\right\rangle_{L^{2}}=1$, and rearranging, we obtain

$$
\begin{aligned}
\left|\alpha-\alpha^{\mathrm{ref}}\right|(t) & \leq|\ell|_{L^{1}}\left(\left|u^{\mathrm{ref}}\right|_{L^{\infty}}(t)+C\left|\alpha-\alpha^{\mathrm{ref}}\right|^{2}\right) \\
& \leq C_{2}\left(E_{0}(1+t)^{-1 / 2}+\left|\alpha-\alpha^{\mathrm{ref}}\right|^{2}\right),
\end{aligned}
$$

yielding (C.8)(vi) provided $\left|\alpha-\alpha^{\mathrm{ref}}\right|$ is sufficiently small. The result then follows by continuity of $\alpha, \alpha^{\text {ref }}$ and smallness of $\alpha$ at $t=0$ for $E_{0}$ small, recalling that $\alpha^{\text {ref }}(0)=$ 0 .

REMARK C.2. As the only bound used on $\ell$ was its $L^{1}$ norm, the proof of Proposition C.1 is easily adapted to the case that $\ell$ is a bounded measure, in particular the case of a phase condition (C.4). This includes also more general cases such as the sum of point measures, leading to a sort of "difference stencil" condition determining shock location.

REMARK C.3. Recalling that $\alpha^{\mathrm{ref}}(t)$ in general decays at most at a subalgebraic rate (see Remark 3.9), we see from (C.8) (iv) that $\alpha$ and $\alpha^{\text {ref }}$ are time-asymptotically equivalent in the sense that $\left|\alpha-\alpha^{\text {ref }}\right|$ decays at a rate faster than the (general) rate of decay of $\left|\alpha^{\mathrm{ref}}\right|$.

Remark C.4. For initial data in addition decaying as $\left|u_{0}(x)\right| \leq C E_{0}(1+|x|)^{-3 / 2}$, it is shown for general systems in $\left[\mathrm{HR}, \mathrm{RZ}\right.$. that $\alpha^{\text {ref }}$ decays at the faster rate

$$
\left|\alpha^{\mathrm{ref}}(t)-\alpha^{\mathrm{ref}}(+\infty)\right| \leq C E_{0}(1+t)^{-1 / 2} .
$$

However, the same analysis yields sharpened bounds on $u^{\text {ref }}$ as well, giving also

$$
\left|u^{\mathrm{ref}}(x, t)\right| \leq C E_{0}(1+t)^{-1} \text { for }|x| \leq \theta t,
$$

$\theta>0$ sufficiently small. Substituting in (C.10), we obtain in place of (C.11) the estimate

$$
\left|\alpha-\alpha^{\mathrm{ref}}\right|(t) \leq C E_{0}(1+t)^{-1}|\ell|_{L^{1}}+C E_{0}(1+t)^{-1 / 2} \int_{|x| \geq \theta t}|\ell(x)| d x+C\left|\alpha-\alpha^{\mathrm{ref}}\right|^{2},
$$

yielding $\left|\alpha-\alpha^{\text {ref }}\right|(t) \leq C E_{0}(1+t)^{-1}$ provided $|\ell(x)| \leq C(1+|x|)^{-3 / 2}$.

Thus, under this strengthened decay requirement on $\ell$, we obtain time-asymptotic equivalence of $\alpha$ and $\alpha^{\text {ref }}$ also in this case. Bound (C.12) is sharp, as can be seen by direct computation on the linear term in (3.19) for data decaying as $(1+|x|)^{-3 / 2}$. (Note that the linear $O\left(E_{0}\right)$ term dominates the nonlinear $O\left(E_{0}^{2}\right)$ term up to any finite time, for $E_{0}$ sufficiently small.)

Conclusions. By comparison with the scheme of Section 3, we find that each of the alternative shock-tracking schemes described in this Appendix, based on localized phase conditions, yields a globally defined solution exhibiting the same rates of decay as the perturbation $u^{\text {ref }}$ defined in Section 3. That is, essentially any tracking scheme based on information that is "local to the shock" in the sense that it is accessible by an inner product with an $L^{1}$ function (resp. bounded measure) $\ell$ yields a convergent system of perturbation equations. Note, further, that the only information used to draw these conclusions consists of estimates on $\left(u^{\text {ref }}, \alpha^{\text {ref }}\right)$ already established in [Z1, MaZ2, MaZ3, MaZ4, HZ, RZ for Evans-stable Lax or undercompressive type shocks of general hyperbolic-parabolic systems. Thus, the conclusions of Proposition C.1 and Remarks 
C.2 C.4 remain valid for Evans-stable Lax or undercompressive shocks of general systems of hyperbolic-parabolic conservation laws 4

An interesting question is whether we could carry out a nonlinear stability analysis for these schemes from first principles rather than by comparison to our existing results. This is particularly intriguing for the case of the pointwise phase condition (C.4), for which resolvent (and thus pointwise Green function) bounds are available through the framework developed in GMWZ. Besides the intrinsic interest of this question, there are real advantages to the scheme based on (C.4) for extension to more complicated situations: for example, the fact that it is local in time (the scheme in Section 3 by contrast involves "memory terms"), and that the phase condition (C.4 makes no reference to the explicit structure of the system.

\section{Appendix D. Miscellaneous estimates.}

D.1. Bounds on e.

Lemma D.1. For some $C>0$ and all $t>0$,

$$
\begin{aligned}
& |e(\cdot, t)|_{L^{\infty}}, \leq C, \\
& \left|e_{y}(\cdot, t)\right|_{L^{p}},\left|e_{t}(\cdot, t)\right|_{L^{p}} \leq C t^{-\frac{1}{2}(1-1 / p)}, \\
& \left|e_{t y}(\cdot, t)\right|_{L^{p}} \leq C t^{-\frac{1}{2}(1-1 / p)-1 / 2}, \\
& \left|e_{y}(y, t)\right|,\left|e_{t}(y, t)\right| \leq C t^{-1 / 2}\left(e^{-\frac{(-y-t)^{2}}{C t}}+e^{-\frac{(-y+t)^{2}}{C t}}\right), \\
& \left|e_{t y}(y, t)\right| \leq C t^{-1}\left(e^{-\frac{(-y-t)^{2}}{C t}}+e^{-\frac{(-y+t)^{2}}{C t}}\right) .
\end{aligned}
$$

Proof. Bound (D.1) follows immediately from definition (3.7). Given (D.4)-(D.5), bounds (D.2)-(D.3) follow as in the heat kernel estimates (2.7)-(2.8). Thus, it remains only to establish (D.4)-(D.5). Differentiating (3.7), we have $e_{y}(y, t)=$ $\left(\frac{1}{u_{+}-u_{-}}\right)\left(\frac{e^{-\frac{(-y-t)^{2}}{4 t}}}{\sqrt{4 \pi t}}-\frac{e^{-\frac{(-y+t)^{2}}{4 t}}}{\sqrt{4 \pi t}}\right)$, yielding (D.4) (i). Differentiating (3.7) with respect to $t$, we obtain

$$
\begin{aligned}
e_{t}(y, t)= & \left(\frac{-1}{2}\right)\left(\frac{e^{-\frac{(-y-t)^{2}}{4 t}}}{\sqrt{4 \pi t}}+\frac{e^{-\frac{(-y+t)^{2}}{4 t}}}{\sqrt{4 \pi t}}\right) \\
& -\left(\frac{t^{-1 / 2}}{2}\right)\left(\frac{(-y-t)}{\sqrt{t}} \frac{e^{-\frac{(-y-t)^{2}}{4 t}}}{\sqrt{4 \pi t}}-\frac{(-y+t)}{\sqrt{t}} \frac{e^{-\frac{(-y+t)^{2}}{4 t}}}{\sqrt{4 \pi t}}\right),
\end{aligned}
$$

\footnotetext{
${ }^{4}$ With the inclusion of additional phase conditions to account for additional degrees of freedom in the time-asymptotic state (see [HZ [RZ]), these methods and estimates extend also to the overcompressive case.
} 
yielding (D.4) (ii) immediately for $t \geq 1$. By the Mean Value Theorem, for $t \leq 1$,

$$
\begin{aligned}
\left|\frac{(-y-t)}{\sqrt{t}} \frac{e^{-\frac{(-y-t)^{2}}{4 t}}}{\sqrt{4 \pi t}}-\frac{(-y+t)}{\sqrt{t}} \frac{e^{-\frac{(-y+t)^{2}}{4 t}}}{\sqrt{4 \pi t}}\right| & =t\left|\int_{-1}^{1} \partial_{z}\left(\frac{z}{\sqrt{t}} \frac{e^{-\frac{z^{2}}{4 t}}}{\sqrt{4 \pi t}}\right)\right|_{z=-y+\theta t} d \theta \mid \\
& \leq 2 C t\left|\partial_{z}\left(\frac{z}{\sqrt{t}} \frac{e^{-\frac{z^{2}}{4 t}}}{\sqrt{4 \pi t}}\right)\right|_{z=-y} \mid \\
& \leq C\left(e^{-\frac{(-y-t)^{2}}{C t}}+e^{-\frac{(-y+t)^{2}}{C t}}\right),
\end{aligned}
$$

which, together with (D.6), yields again (D.4) (ii). Estimate (D.5) is found similarly. Note that we have taken crucial account of cancellation in the small time estimates of $e_{t}, e_{t y}$.

REMARK D.2. For $t \leq 1$, a calculation analogous to (D.7) yields $\left|e_{y}(y, t)\right| \leq C e^{-\frac{(y+a-t)^{2}}{M t}}$, and thus $|e(\cdot, s)|_{L^{1}} \rightarrow 0$ as $s \rightarrow 0$.

D.2. Bounds on $e^{\varepsilon}$.

Lemma D.3. For some $C>0$, all $0<\varepsilon \leq 1$, and all $t>0$,

$$
\begin{aligned}
& \left|e^{\varepsilon}(\cdot, t)\right|_{L^{\infty}}, \leq C / \varepsilon \\
& \left|e^{\varepsilon}{ }_{y}(\cdot, t)\right|_{L^{p}} \leq(C / \varepsilon) t^{-\frac{1}{2}(1-1 / p)} \\
& \left|e^{\varepsilon}{ }_{t}(\cdot, t)\right|_{L^{p}} \leq C t^{-\frac{1}{2}(1-1 / p)} \\
& \left|e^{\varepsilon}{ }_{t y}(\cdot, t)\right|_{L^{p}} \leq C t^{-\frac{1}{2}(1-1 / p)-1 / 2}, \\
& \left|e^{\varepsilon}{ }_{y}(y, t)\right| \leq(C / \varepsilon) t^{-1 / 2}\left(e^{-\frac{(-y-t)^{2}}{C t}}+e^{-\frac{(-y+t)^{2}}{C t}}\right), \\
& \left|e^{\varepsilon}{ }_{t}(y, t)\right| \leq C t^{-1 / 2}\left(e^{-\frac{(-y-t)^{2}}{C t}}+e^{-\frac{(-y+t)^{2}}{C t}}\right), \\
& \left|e^{\varepsilon}{ }_{t y}(y, t)\right| \leq C t^{-1}\left(e^{-\frac{(-y-t)^{2}}{C t}}+e^{-\frac{(-y+t)^{2}}{C t}}\right) .
\end{aligned}
$$

Proof. Bounds (D.8), (D.9), and (D.12) follow exactly as in the $\varepsilon$-independent case. Bound (D.10) follows immediately provided that we can establish (D.13), as we now do. Differentiating (B.6) with respect to $t$, we obtain

$$
\begin{aligned}
e_{t}^{\varepsilon}(y, t)= & \left(\frac{-1}{2}\right)\left(\frac{e^{-\frac{(-y-\varepsilon t)^{2}}{4 t}}}{\sqrt{4 \pi t}}+\frac{e^{-\frac{(-y+\varepsilon t)^{2}}{4 t}}}{\sqrt{4 \pi t}}\right) \\
& -\left(\frac{t^{-1 / 2}}{2 \varepsilon}\right)\left(\frac{(-y-t)}{\sqrt{t}} \frac{e^{-\frac{(-y-\varepsilon t)^{2}}{4 t}}}{\sqrt{4 \pi t}}-\frac{(-y+\varepsilon t)}{\sqrt{t}} \frac{e^{-\frac{(-y+t)^{2}}{4 t}}}{\sqrt{4 \pi t}}\right),
\end{aligned}
$$


yielding (D.13) immediately for $t \geq \varepsilon^{-2}$. By the Mean Value Theorem, for $t \leq \varepsilon^{-2}$,

$$
\begin{aligned}
\left|\frac{(-y-\varepsilon t)}{\sqrt{t}} \frac{e^{-\frac{(-y-\varepsilon t)^{2}}{4 t}}}{\sqrt{4 \pi t}}-\frac{(-y+\varepsilon t)}{\sqrt{t}} \frac{e^{-\frac{(-y+\varepsilon t)^{2}}{4 t}}}{\sqrt{4 \pi t}}\right| & =\varepsilon t\left|\int_{-1}^{1} \partial_{z}\left(\frac{z}{\sqrt{t}} \frac{e^{-\frac{z^{2}}{4 t}}}{\sqrt{4 \pi t}}\right)\right|_{z=-y+\theta \varepsilon t} d \theta \mid \\
& \leq 2 C \varepsilon t\left|\partial_{z}\left(\frac{z}{\sqrt{t}} \frac{e^{-\frac{z^{2}}{4 t}}}{\sqrt{4 \pi t}}\right)\right|_{z=-y} \mid \\
& \leq C \varepsilon\left(e^{-\frac{(-y-\varepsilon t)^{2}}{C t}}+e^{-\frac{(-y+\varepsilon t)^{2}}{C t}}\right),
\end{aligned}
$$

which, together with (D.15), yields again (D.13). Bounds (D.11) and (D.14) follow similarly.

D.3. Bounds on $R^{\varepsilon}$.

Lemma D.4. For $\mathcal{K} f:=\int_{\mathbb{R}} K(x, y) f(y) d y$ and any $1 \leq p \leq \infty$,

$$
\begin{gathered}
|\mathcal{K} f|_{L^{p}} \leq \sup _{y}|K(\cdot, y)|_{L^{p}}|f|_{L^{1}}, \\
|\mathcal{K}|_{L^{p} \rightarrow L^{p}} \leq \max \left\{\sup _{x}|K(x, \cdot)|_{L^{1}}, \sup _{y}|K(\cdot, y)|_{L^{1}}\right\} .
\end{gathered}
$$

Proof. By the triangle inequality,

$$
\left|\int_{\mathbb{R}} K(\cdot, y) f(y) d y\right|_{L^{p}(x)} \leq \int_{\mathbb{R}}|K(\cdot, y)|_{L^{p}}|f(y)| d y \leq \sup _{y}|K(\cdot, y)|_{L^{p}}|f|_{L^{1}},
$$

establishing (D.17). This yields also (D.18) in case $p=1$. Likewise,

$$
\left|\int_{\mathbb{R}} K(x, y) f(y) d y\right| \leq \int_{\mathbb{R}}|K(x, y)| d y|f|_{L^{\infty}} \leq \sup _{x}|K(x, \cdot)|_{L^{1}}|f|_{L^{\infty}},
$$

establishing (D.18) for $p=\infty$. For general $p$, (D.18) then follows by the Riesz-Thorin Interpolation Theorem.

Lemma D.5. For some $C>0$, all $0<\varepsilon \leq 1$, and all $t>0$,

$$
\begin{aligned}
& \sup _{y}\left|R^{\varepsilon}(\cdot, t ; y)\right|_{L^{p}(x)}, \quad \sup _{x}\left|R^{\varepsilon}(x, t ; \cdot)\right|_{L^{p}(y)} \leq C t^{-\frac{1}{2}(1-1 / p)}, \\
& \sup _{y}\left|R_{y}^{\varepsilon}(\cdot, t ; y)\right|_{L^{p}(x)}, \quad \sup _{x}\left|R_{y}^{\varepsilon}(x, t ; \cdot)\right|_{L^{p}(y)} \leq C t^{-\frac{1}{2}(1-1 / p)-\frac{1}{2}} .
\end{aligned}
$$

Proof. From $\left(\bar{u}^{\varepsilon}\right)^{\prime} \sim \varepsilon^{2} e^{-\theta \varepsilon|x|}$, we obtain

$$
\begin{aligned}
R^{\varepsilon}(x, t ; y) & =\frac{1}{2 \varepsilon} x\left(\bar{u}^{\varepsilon}\right)^{\prime}(x) \int_{0}^{1}\left(\frac{e^{-\frac{(\theta x-y-\varepsilon t)^{2}}{4 t}}}{\sqrt{4 \pi t}}-\frac{e^{-\frac{(\theta x-y+\varepsilon t)^{2}}{4 t}}}{\sqrt{4 \pi t}}\right) d \theta \\
& \leq C e^{-\theta \varepsilon|x|} \int_{0}^{1}\left(\frac{e^{-\frac{(\theta x-y-\varepsilon t)^{2}}{4 t}}}{\sqrt{4 \pi t}}+\frac{e^{-\frac{(\theta x-y+\varepsilon t)^{2}}{4 t}}}{\sqrt{4 \pi t}}\right) d \theta
\end{aligned}
$$

from which we obtain immediately $\left|R^{\varepsilon}\right|_{L^{\infty}} \leq C t^{-1 / 2}$, and, bounding $C e^{-\theta \varepsilon|x|}$ by $C$,

$$
\sup _{x}\left|R^{\varepsilon}\right|_{L^{p}(y)} \leq C t^{-\frac{1}{2}(1-1 / p)}
$$

for any $p$. 
Bounding the integral on the right-hand side by $C_{1} t^{-1 / 2}$ and the $L^{1}(x)$ norm of $C e^{-\theta \varepsilon|x|}$ by $C_{2} / \varepsilon$, we find $\sup _{y}\left|R^{\varepsilon}\right|_{L^{1}(x)} \leq C_{2} t^{-1 / 2} / \varepsilon \leq C$ for $t \geq \varepsilon^{-2}$. For $t \leq \varepsilon^{-2}$, on the other hand, we may estimate the integral (the middle displayed term in the first equality) instead, using the Mean Value Theorem, as

$$
\begin{aligned}
\int_{0}^{1}\left(\frac{e^{-\frac{(\theta x-y-\varepsilon t)^{2}}{4 t}}}{\sqrt{4 \pi t}}-\frac{e^{-\frac{(\theta x-y+\varepsilon t)^{2}}{4 t}}}{\sqrt{4 \pi t}}\right) d \theta & \leq\left.\int_{0}^{1}(2 \varepsilon t) \partial_{z}\left(\frac{e^{-\frac{(\theta x-y-z)^{2}}{4 t}}}{\sqrt{4 \pi t}}\right)\right|_{z=z_{*} \in[-\varepsilon t, \varepsilon t]} d \theta \\
& \leq(2 \varepsilon t) \int_{0}^{1} C t^{-1} d \theta \leq C \varepsilon
\end{aligned}
$$

to again obtain $\sup _{y}\left|R^{\varepsilon}\right|_{L^{1}(x)} \leq C_{2} \varepsilon / \varepsilon \leq C$. The bounds on $\sup _{y}\left|R^{\varepsilon}\right|_{L^{p}(x)}$ then follow by Hölder interpolation between the $L^{1}$ and $L^{\infty}$ bounds, verifying (D.19) Similar computations yield (D.20).

D.4. Euler-Lagrange equations for least squares. Setting $E(\alpha):=\frac{1}{2}|u|_{L^{2}}^{2}=\frac{1}{2} \mid \tilde{u}(\cdot+$ $\alpha, t)-\left.\bar{u}(\cdot)\right|_{L^{2}} ^{2}$ and differentiating, we have

$$
\frac{d E}{d \alpha}=\left\langle\tilde{u}(\cdot+\alpha, t)-\bar{u}(\cdot), \tilde{u}^{\prime}(\cdot+\alpha, t)\right\rangle_{L^{2}}=\left\langle u, \bar{u}^{\prime}+u^{\prime}\right\rangle_{L^{2}}=\left\langle u, \bar{u}^{\prime}\right\rangle_{L^{2}},
$$

where, in the final equality, we have used $\left\langle u, u^{\prime}\right\rangle_{L^{2}}=\int_{-\infty}^{+\infty}\left(u^{2} / 2\right)^{\prime}(x) d x=0$ for $u \in H^{1}$.

\section{REFERENCES}

[AGJ] J. Alexander, R. Gardner and C.K.R.T. Jones, A topological invariant arising in the analysis of traveling waves, J. Reine Angew. Math. 410 (1990) 167-212. MR1068805 (92d:58028)

[BHZ] B. Barker, J. Humpherys, and K. Zumbrun, One-dimensional stability of parallel shock layers in isentropic magnetohydrodynamics, to appear, J. Diff. Eq.

[BHRZ] B. Barker, J. Humpherys, , K. Rudd, and K. Zumbrun, Stability of viscous shocks in isentropic gas dynamics, Comm. Math. Phys. 281 (2008), no. 1, 231-249. MR2403609 (2009c:35286)

[BDG] T.J. Bridges, G. Derks, and G. Gottwald, Stability and instability of solitary waves of the fifth-order KdV equation: a numerical framework. Phys. D 172 (2002), no. 1-4, 190-216. MR 1946769 (2004i:37148)

[CHNZ] N. Costanzino, J. Humpherys, T. Nguyen, and K. Zumbrun, Spectral stability of noncharacteristic isentropic Navier-Stokes boundary layers, Arch. Ration. Mech. Anal. 192 (2009), 537-587. MR2505363

[Br] L. Q. Brin, Numerical testing of the stability of viscous shock waves. Math. Comp. 70 (2001) no. 235, 1071-1088. MR1710652 (2001j:65118)

[BrZ $\quad$ L. Brin and K. Zumbrun, Analytically varying eigenvectors and the stability of viscous shock waves. Seventh Workshop on Partial Differential Equations, Part I (Rio de Janeiro, 2001). Mat. Contemp. 22 (2002), 19-32. MR.1965784 (2004c:15012)

[BeSZ] M. Beck, B. Sandstede, and K. Zumbrun, Nonlinear stability of time-periodic viscous shocks, Arch. Ration. Mech. Anal. 196 (2010), 1011-1076. MR2644447

[FS1] H. Freistühler and P. Szmolyan, Spectral stability of small shock waves, Arch. Ration. Mech. Anal. 164 (2002) 287-309. MR.1933630 (2003j:35273)

[FS2] H. Freistühler and P. Szmolyan. Spectral stability of small-amplitude viscous shock waves in several space dimensions, Arch. Ration. Mech. Anal. 195 (2010), 353-373. MR.2592280

[GZ] R. Gardner and K. Zumbrun, The Gap Lemma and geometric criteria for instability of viscous shock profiles. Comm. Pure Appl. Math. 51 (1998), no. 7, 797-855. MR1617251 (99c:35152)

[G] J. Goodman, Remarks on the stability of viscous shock waves, in: Viscous profiles and numerical methods for shock waves (Raleigh, NC, 1990), 66-72, SIAM, Philadelphia, PA, 1991. MR 1142641

[GSZ] J. Goodman, A. Szepessy, and K. Zumbrun, A remark on the stability of viscous shock waves, SIAM J. Math. Anal. 25 (1994) 1463-1467. MR.1302156 (95i:35182) 
[GMWZ] O. Guès, G. Métivier, M. Williams, and K. Zumbrun. Existence and stability of multidimensional shock fronts in the vanishing viscosity limit, Arch. Ration. Mech. Anal. 175 (2005), no. 2, 151-244. MR2118476 (2005k:35273)

[GMWZ2] O. Guès, G. Métivier, M. Williams, and K. Zumbrun. Existence and stability of noncharacteristic hyperbolic-parabolic boundary-layers for the compressible Navier-Stokes and viscous MHD equations, Arch. Ration. Mech. Anal. 197 (2010), no. 1, 1-87.

[HZ] P. Howard and K. Zumbrun, Stability of undercompressive viscous shock waves, J. Differential Equations 225 (2006), no. 1, 308-360; preprint 2004. MR2228699(2007d:35185)

[HR] P. Howard and M. Raoofi, Pointwise asymptotic behavior of perturbed viscous shock profiles, Adv. Differential Equations (2006) 1031-1080. MR2263670(2007i:35161)

[HRZ] P. Howard, M. Raoofi, and K. Zumbrun, Sharp pointwise bounds for perturbed viscous shock waves, J. Hyperbolic Differ. Equ. 3 (2006) 297-373; preprint 2005. MR 2229858(2006m:35235)

[HLZ] J. Humpherys, O. Lafitte, and K. Zumbrun, Stability of isentropic Navier-Stokes shocks in the high-Mach number limit, Comm. Math. Phys. 293 (2010), 1-36; published online, Sept. 2009. MR2563797 (2010i:76085)

[HLyZ] J. Humpherys, G. Lyng, and K. Zumbrun, Spectral stability of ideal-gas shock layers, Arch. Ration. Mech. Anal. 194 (2009), 1029-1079. MR2563632

[HuZ] J. Humpherys and K. Zumbrun, Spectral stability of small-amplitude shock profiles for dissipative symmetric hyperbolic-parabolic systems. Z. Angew. Math. Phys. 53 (2002) 20-34. MR.1889177 (2003b:35133)

[HuZ2] J. Humpherys and K. Zumbrun, An efficient shooting algorithm for Evans function calculations in large systems, Phys. D 220 (2006), no. 2, 116-126. MR.2253406 (2007e:35006)

[JZ1] M. Johnson and K. Zumbrun, Nonlinear stability of periodic traveling wave solutions of viscous conservation laws in dimensions one and two, to appear, SIAM J. Math. Anal.

[JZ2] M. Johnson and K. Zumbrun, Nonlinear stability of periodic traveling wave solutions of systems of viscous conservation laws in the generic case, J. Diff. Eq. 249 (2010), 1213-1240. MR2652171

[JZN] M. Johnson, K. Zumbrun, and P. Noble, Nonlinear stability of viscous roll waves, to appear, SIAM J. Math. Anal.

[Liu85] T.P. Liu, Nonlinear stability of shock waves for viscous conservation laws, Mem. Amer. Math. Soc. 56 (1985), no. 328, v+108 pp. MR791863 (87a:35127)

[Liu97] T.P. Liu, Pointwise convergence of shock waves for viscous conservation laws, Comm. Pure Appl. Math. 50 (1997), no. 11, 1113-1182. MR.1470318 (98j:35121)

[LY] T.P. Liu and S.H. Yu, Propagation of a stationary shock layer in the presence of a boundary, Arch. Rational Mech. Anal. 139 (1997), no. 1, 57-82. MR.1475778 (99b:35137)

[LZ2] T.P. Liu and Y. Zeng, Time-asymptotic behavior of wave propagation around a viscous shock profile, Comm. Math. Phys. 290 (2009), 23-82. MR2520507 (2010j:35325)

[LRTZ] G. Lyng, M. Raoofi, B. Texier, and K. Zumbrun, Pointwise Green function bounds and stability of combustion waves, J. Differential Equations 233 (2007), no. 2, 654-698. MR.2292522 (2007m:35147)

[LZu] T.P. Liu and K. Zumbrun, On nonlinear stability of general undercompressive viscous shock waves, Commun. Math. Phys. 174 (1995) 319-345. MR1362168 (97g:35110)

[MaZ1] C. Mascia and K. Zumbrun, Pointwise Green's function bounds and stability of relaxation shocks, Indiana Univ. Math. J. 51 (2002), no. 4, 773-904. MR1947862 (2003k:35151)

[MaZ2] C. Mascia and K. Zumbrun, Stability of small-amplitude shock profiles of symmetric hyperbolic-parabolic systems, Comm. Pure Appl. Math. 57 (2004), no. 7, 841-876; preprint 2001. MR2044067 (2005e:35022)

[MaZ3] C. Mascia and K. Zumbrun, Pointwise Green function bounds for shock profiles of systems with real viscosity. Arch. Ration. Mech. Anal. 169 (2003), no. 3, 177-263; preprint 2002. MR2004135 (2004h:35137)

[MaZ4] C. Mascia and K. Zumbrun, Stability of large-amplitude viscous shock profiles of hyperbolicparabolic systems, Arch. Ration. Mech. Anal. 172 (2004), no. 1, 93-131; preprint 2003. MR2048568 (2005d:35166)

[N] K. Nishihara, A note on the stability of travelling wave solutions of Burgers' equation (English) Japan. J. Appl. Math. 2 (1985), no. 1, 27-35. MR839318 (87j:35335b) 
[PZ] R. Plaza and K. Zumbrun, An Evans function approach to spectral stability of smallamplitude shock profiles, J. Disc. and Cont. Dyn. Sys. 10 (2004), 885-924. MR2073940 (2005d:35169)

[R] M. Raoofi, $L^{p}$ asymptotic behavior of perturbed viscous shock profiles, J. Hyperbolic Differ. Equ. 2 (2005), no. 3, 595-644; preprint 2004. MR2172698 (2006j:35163)

[RZ] M. Raoofi and K. Zumbrun, Stability of undercompressive viscous shock profiles of hyperbolicparabolic systems, J. Differential Equations 246 (2009) 1539-1567. MR.2488696|(2010a:35107)

[S] D. Sattinger, On the stability of waves of nonlinear parabolic systems, Adv. Math. 22 (1976) 312-355. MR0435602 (55:8561)

[SX] A. Szepessy and Z. Xin, Nonlinear stability of viscous shock waves, Arch. Ration. Mech. Anal. 122 (1993) 53-103. MR.1207241 (93m:35125)

[SS] B. Sandstede and A. Scheel, Hopf bifurcation from viscous shock waves, SIAM J. Math. Anal. 39 (2008) 2033-2052. MR2390324 (2009f:35218)

[TZ1] B. Texier and K. Zumbrun, Relative Poincaré-Hopf bifurcation and galloping instability of traveling waves, Methods Appl. Anal. 12 (2005), no. 4, 349-380. MR2258314 (2007h:37118)

[TZ2] B. Texier and K. Zumbrun, Galloping instability of viscous shock waves, Physica D. 237 (2008) 1553-1601. MR.2454606 (2009h:35271)

[TZ3] B. Texier and K. Zumbrun, Hopf bifurcation of viscous shock waves in gas dynamics and MHD, Arch. Ration. Mech. Anal. 190 (2008) 107-140. MR2434902 (2009g:35239)

[TZ4] B. Texier and K. Zumbrun, Transition to longitudinal instability of detonation waves is generically associated with Hopf bifurcation to time-periodic galloping solutions, to appear, Comm. Math. Phys.; preprint (2008).

[Z1] K. Zumbrun, Refined wave-tracking and stability of viscous Lax shocks, Methods Appl. Anal. 7 (2000) 747-768; preprint, 1999. MR.1868555 (2002j:35211)

[Z2] K. Zumbrun, Planar stability criteria for multidimensional viscous shock waves, Hyperbolic systems of balance laws, 229-326, Lecture Notes in Math., 1911, Springer, Berlin, 2007. (Lectures given at the C.I.M.E. Summer School held in Cetraro, July 14-21, 2003; preprint, 2003.) MR2348937 (2008k:35315)

[Z3] K. Zumbrun, Formation of diffusion waves in a scalar conservation law with convection, Trans. Amer. Math. Soc. 347 (1995), no. 3, 1023-1032. MR.1283568 (95e:35124)

[Z4] K. Zumbrun, Stability of large-amplitude shock waves of compressible Navier-Stokes equations, with an appendix by Helge Kristian Jenssen and Gregory Lyng, in Handbook of mathematical fluid dynamics. Vol. III, 311-533, North-Holland, Amsterdam, 2004. MR2099037 (2006f:35229)

[Z5] K. Zumbrun, Conditional stability of unstable viscous shocks, J. Differential Equations 247 (2009), no. 2, 648-671. MR2523696

[Z6] K. Zumbrun, Multidimensional stability of planar viscous shock waves, Advances in the theory of shock waves, 307-516, Progr. Nonlinear Differential Equations Appl., 47, Birkhäuser Boston, Boston, MA, 2001. MR.1842778 (2002k:35200)

[Z7] K. Zumbrun, Conditional stability of unstable viscous shock waves in compressible gas dynamics and MHD, to appear, Arch. for Ration. Mech. Anal.; preprint (2009).

[ZH] K. Zumbrun and P. Howard, Pointwise semigroup methods and stability of viscous shock waves. Indiana Mathematics Journal 47 (1998), 741-871; Errata, Indiana Univ. Math. J. 51 (2002), no. 4, 1017-1021. MR.1665788 (99m:35157) MR.1947866 (2004a:35155)

[ZS] K. Zumbrun and D. Serre, Viscous and inviscid stability of multidimensional planar shock fronts, Indiana Univ. Math. J. 48 (1999) 937-992. MR.1736972 (2001h:35122) 